\title{
Búsquedas de Materia Oscura Supersimétrica en el LHC
}

\author{
Juan Carlos Sanabria \\ Departamento de Física, Universidad de Los Andes, Colombia
}

\begin{abstract}
Resumen
La composición microscópica de la materia oscura y la observación directa o indirecta de su presencia, más allá de sus efectos gravitacionales, representa uno de los problemas más urgentes que enfrentan la Física de Partículas, la Astrofísica y la Cosmología. Desde hace varios años se han llevado a cabo búsquedas experimentales de diversa índole, sin ningún resultado concluyente. Toda la evidencia indica que los constituyentes microscópicos de la materia oscura no pueden pertenecer al espectro de partículas del Modelo Estándar. Varios modelos teóricos proponen candidatos a ser los constituyentes fundamentales de este tipo de materia; entre ellos, algunas versiones de modelos supersimétricos, en las cuales los neutralinos más ligeros son estables, masivos, neutros y sin carga de color, haciéndolos excelentes candidatos. En los experimentos del LHC se han llevado a cabo búsquedas exhaustivas de partículas supersimétricas durante las primeras corridas a energías de 7 y $8 \mathrm{TeV}$, sin ningún resultado positivo. Estas búsquedas se han concentrado en canales de producción fuertes, con gluinos y s-quarks en el estado final. Dados los resultados negativos, es imperativo llevar a cabo búsquedas detalladas en canales de producción electro-débiles, en los cuales las partículas supersimétricas del estado final deberán ser más ligeras y, por tanto, tener mayor probabilidad de ser observadas, incluyendo los neutralinos. Los canales de producción de materia supersimétrica mediados por la fusión de bosones vectoriales son, hoy por hoy, los más prometedores, desde este punto de vista. En este artículo se presenta una revisión de las búsquedas de materia oscura en las primeras corridas del LHC, haciendo énfasis en los canales de fusión de bosones vectoriales y se presentan estrategias para llevar a cabo estas búsquedas, en la próxima corrida, a una energía de $14 \mathrm{TeV}$.
\end{abstract}

\section{Search for Supersymmetric Dark Matter in the LHC}

\begin{abstract}
The microscopic composition and the direct or indirect observation of dark matter, other than trough its gravitational effects, is currently one of the most urgent and important problems in particle physics, astrophysics and cosmology. Since several years ago, different kinds of experimental searches have been conducted without any conclusive results. All the evidence shows that the microscopic constituents of dark matter cannot belong to the particle spectrum of the Standard Model. Several other theoretical models provide candidates to be the constituents of this kind of matter, among them some versions of supersymmetric models, in which the lightest neutralinos are stable, neutral, massive and do not have color charge, making them excellent candidates. The LHC experiments have conducted thorough searches of supersymmetric particles during the first runs of the LHC at 7 and $8 \mathrm{TeV}$, without any positive result. These searches have been concentrated in strong production channels, with gluinos and s-quarks in the final state. Given the negative results, it is necessary to perform detailed searches in electro-weak channels in which the supersymmetric particles in the final state are expected to be lighter, and therefore, have higher probability to be produced, including the neutralinos. The supersymmetric production channels mediated by vector boson fusion are the most promising ones. In this article, a review of the dark matter searches performed during the first runs of the LHC are presented, making emphasis on the vector boson fusion channels, as well as the strategies for the searches to be performed in the next run, at $14 \mathrm{TeV}$.
\end{abstract}

\section{Introduction}

En 2012, con el descubrimiento del bosón de Higgs en los experimentos CMS y ATLAS en el acelerador LHC del laboratorio CERN [1, 2], se cerró una página importante en la historia de la Física. Esta era la última partícula por descubrir del Modelo Estándar de Partículas e Interacciones (SM, Standard Model), aún cuando paradójicamente era, tal vez, la más importante dado el papel que juega al proveer términos de masa para los demás campos $\mathrm{y}$, de paso, para restablecer la renormalizabilidad de todo el modelo. Si bien el
SM representa una excelente versión de las leyes de la física, al nivel más elemental, continúa teniendo algunos problemas a nivel teórico que tienen que ver con su comportamiento matemático a muy altas energías y con su incapacidad para dar explicaciones microscópicas satisfactorias a los dos problemas pendientes más importantes de la Astrofísica y de la Cosmología: la naturaleza de la materia oscura y de la energía oscura. Todo indica que el SM es una teoría efectiva que funciona muy bien a la escala electro-débil, es decir, a energías que van hasta los $\mathrm{TeVs}$, pero que 
tiene un comportamiento matemático insatisfactorio a escalas de energía superiores, como lo es la escala de Gran Unificación $\left(\sim 10^{13} \mathrm{TeV}\right)$. Durante los últimos 50 años, se ha llevado a cabo un gran esfuerzo por construir modelos teóricos que vayan más allá del SM, pero que, por supuesto, lo incluyan y reproduzcan sus resultados a la escala electro-débil (BSM, Beyond Standard Model). Todos estos modelos predicen la existencia de una gama de nuevas partículas elementales muy masivas que, por lo tanto, solo son observables en ambientes con altas densidades de energía, como lo fueron las épocas tempranas del universo y como lo serían las colisiones entre protones en aceleradores como el LHC. Estas partículas, dada su alta masa, tendrían tiempos de vida muy cortos y habrían decaído muy pronto después del Big-Bang, contribuyendo a la composición actual de la materia bariónica y leptónica, y a la radiación cósmica de fondo del universo. Sin embargo, en ciertas versiones de estos modelos, algunas de estas partículas son estables, a pesar de ser masivas y, además, son el producto final de las cadenas de decaimiento de partículas de mayor masa. En consecuencia, enormes cantidades de ellas deberían haberse acumulado después del Big-Bang y continuar deambulando por el cosmos aún hoy en día. Dada la alta masa de estas partículas y a su gran cantidad, sus efectos gravitacionales deberían ser manifiestos, pero también, dado que aún no las hemos descubierto en la Tierra, deberían interactuar muy poco con la materia ordinaria que conocemos. La materia ordinaria tiene una alta interacción electromagnética a nivel atómico y una alta interacción fuerte a nivel nuclear. Es de esperarse, por tanto, que estas partículas interactúen gravitacionalmente, y a lo sumo, débilmente (WIMP, Weakly Interacting Massive Particle). Modelos teóricos BSM, como por ejemplo, los modelos supersimétricos (SUSY, Supersymmetry) y los modelos con dimensiones espaciales extra (ED, Extra Dimensions) proponen partículas que cumplen con estas condiciones básicas. Los efectos gravitacionales de estas partículas deberían ser notorios, proporcionando una respuesta al problema de la composición microscópica de la materia oscura; una forma de materia de la cual solo conocemos sus efectos gravitacionales a gran escala.

Las primeras evidencias de una forma de materia cuya naturaleza desconocemos, pero que tiene efectos gravitacionales notorios, provienen de las observaciones astronómicas de Fritz Zwicky, quien, en 1933, llevó a cabo un estudio detallado de la dinámica del cúmulo de galaxias Coma, conformado por unas 2000 galaxias y localizado a 300 millones de años luz de la Tierra. Usando la técnica de corrimiento Doppler de la luz recibida de cada galaxia para determinar su velocidad, Zwicky estimó la masa contenida en el cúmulo. El resultado indicaba que esta masa era mucho mayor a la cantidad de materia visible, unas 500 veces mayor [3] (actualmente se estima que es sólo 200 veces mayor). Sinclair Smith, en 1936, obtuvo resultados similares para el cúmulo de
Virgo [4]. Zwicky continuó sus estudios a nivel galáctico, midiendo velocidades de rotación de estrellas orbitando alrededor del núcleo de galaxias espirales. Sus resultados indicaban que, también en este caso, la dinámica de estas estrellas estaba alterada con respecto a lo que se esperaría, dada la cantidad de materia observada. Si se usa gravitación newtoniana para calcular las velocidades de rotación de las estrellas, no se obtienen los resultados medidos, a menos que se considere la presencia de un halo de materia que no observamos, pero que ejerce una influencia gravitacional importante [5]. Toda la evidencia recolectada por Zwicky parecía indicar que en el universo había grandes cantidades de materia oscura.

En galaxias espirales, la gran mayoría de estrellas visibles están concentradas en un núcleo central. Las demás estrellas y gases orbitan alrededor del núcleo en un disco exterior. Las estrellas que están en este disco deberían seguir patrones de movimiento similares a los que siguen los planetas que orbitan alrededor del Sol. La velocidad de rotación de una estrella es de la forma:

$$
v(r)=\sqrt{\frac{G M(r)}{r}},
$$

donde

$$
M(r)=4 \pi \int_{0}^{r} \rho\left(r^{\prime}\right) r^{\prime 2} d r^{\prime}
$$

es la masa incluida al interior de la órbita y $\rho(r)$ es el perfil de densidad de masa de la galaxia. En consecuencia, si consideramos que la gran mayoría de la masa de la galaxia está acumulada en su núcleo, y despreciamos la masa que está en el disco, entonces $v(r) \propto 1 / \sqrt{r}$, en la región del disco. Por el contrario, las estrellas al interior del núcleo galáctico deberían poseer velocidades que incrementan con la distancia al centro. El resultado debería ser una curva de velocidad rotacional que aumenta desde cero en el centro galáctico y que llega a un máximo en la frontera del núcleo, para después disminuir en la región del disco (ver Figura 1). Sin embargo, esto no fue lo que observó Zwicky; lo que él observó fue una curva que incrementa al interior del núcleo, para después hacerse constante en la región del disco galáctico (ver Figura 1). Este tipo de estudios fueron llevados a cabo hasta la década de 1970, usando la técnica de corrimiento Doppler para la luz visible de estrellas y de nubes de gas luminoso al interior de las galaxias. El uso de radio-telescopios para medir corrimientos Doppler de la radiación emitida con una longitud de onda de $21 \mathrm{~cm}$ por nubes de hidrógeno, orbitando más allá de los límites visibles de las galaxias, permitieron extender las curvas de velocidad de rotación [6, 7]. Estos estudios mostraban que hasta los límites detectables de las galaxias, las curvas continúan siendo planas, aun a grandes distancias del núcleo. Una 
forma de explicar estos resultados es asumiendo que hay una cantidad apreciable de materia invisible, que se esparce en forma de halo hasta los confines de la galaxia, conteniendo de 5 a 10 veces más masa que la que observamos, afectando la dinámica de la materia visible. Este halo debería poseer un perfil de densidad $\rho(r) \propto 1 / r^{2}$, de tal forma que $M(r) \propto r$ y por tanto $v(r) \propto$ const.

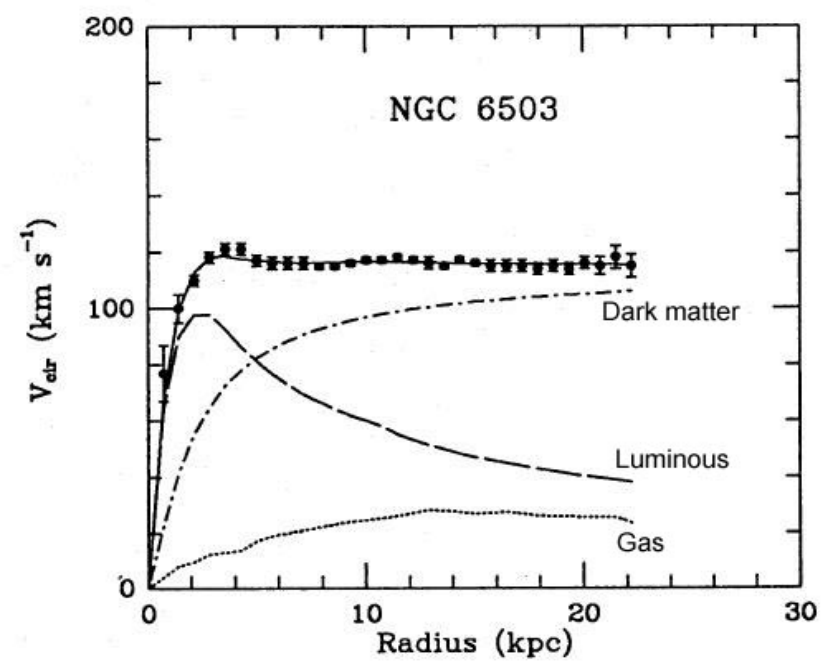

Figura 1: Curva de rotación para la galaxia espiral NGC 6503. Los datos son consistentes con la presencia de materia luminosa, de gas y de materia oscura, como se muestra en la gráfica (tomada de la referencia [6]).

Nuestra galaxia, la Vía Láctea, debería contener una cantidad apreciable de materia oscura en forma de halo permeando la región visible e incluyendo al Sistema Solar. El movimiento de las estrellas que se encuentran fuera del plano galáctico; de los cúmulos globulares; de galaxias satélites como las Nubes de Magallanes; al igual que la interacción gravitacional con nuestra galaxia hermana Andrómeda, permiten estimar la cantidad de masa en el halo que resulta ser unas 10 veces mayor que la masa de la materia luminosa que observamos [8-11].

El caso de las galaxias elípticas, en el cual es mucho más difícil hacer curvas de velocidad de rotación, dado que no hay un plano bien definido en el cual las estrellas estén rotando, los estudios de ensanchamiento de las líneas espectrales de la luz emitida por las estrellas, nos dan una medida de las velocidades estelares al interior de la galaxia. El teorema Virial nos permite establecer una relación entre la energía cinética total del sistema y la energía potencial que, en este caso es gravitacional. De esta forma podemos determinar la energía potencial gravitacional y, por tanto, la masa total de la galaxia. Nuevamente, este tipo de estudios muestran un exceso de materia oscura que es 5 a 10 veces mayor que el de la materia luminosa [12]. Sin embargo, estudios muy detallados de las distribuciones de velocidad de nebulosas planetarias (las nubes de gas emitidas por estrellas que están muriendo), en galaxias elípticas cercanas, muestran que algunas de ellas parecieran tener muy poca materia oscura, o no tenerla. Este es un resultado sorprendente que proyecta una sombra de duda sobre algunos preceptos relacionados con las teorías de formación y de estructura de las galaxias, a la luz de la existencia de materia oscura [13]. Otro resultado, igualmente intrigante, es la existencia de galaxias oscuras, que contienen muy pocas estrellas, pero poseen grandes cantidades de materia oscura. Un ejemplo de este tipo de galaxias es el objeto denominado VIRGOHI21, descubierto en 2005 por medio de la detección de ondas de radio de $21 \mathrm{~cm}$ emitidas por nubes de hidrógeno [14]. Esta galaxia oscura parece tener una masa de 10,000 millones de soles y extenderse por una región del espacio de 50,000 años luz.

Los estudios de la dinámica de los cúmulos y supercúmulos de galaxias, más allá de las observaciones iniciales llevadas a cabo por Zwicky y Smith, han mostrado que, aun incluyendo la masa proveniente del gas ionizado presente en el espacio intergaláctico, la masa visible o detectable de estos objetos no alcanza un décimo de la masa que se deduce de su comportamiento dinámico $[15,16]$.

Una evidencia bastante directa de la presencia de materia oscura en el universo proviene del fenómeno conocido como lente gravitacional. Según la teoría de la Relatividad General, un haz de luz es desviado al pasar cerca de una fuente intensa de campo gravitacional. Por lo tanto, un cuerpo muy masivo que esté en el camino entre un objeto luminoso y un observador, actuará como un lente convergente, magnificando la imagen del objeto. Si el objeto, el cuerpo masivo y el observador están alineados, la luz del objeto se desviará produciendo una imagen en forma de anillo desde el punto de vista del observador. Si el alineamiento no es perfecto, se observarán varias copias del objeto, distorsionadas en forma de arcos, como las observadas por Walsh, Carswell y Weymann en 1979 para el qúasar 0957+561 [17]. El ejemplo por excelencia del fenómeno de lente gravitacional es el cúmulo Abell 2218, en la constelación del Dragón, que muestra imágenes distorsionadas en forma de arcos de más de 100 galaxias que están en un cúmulo mucho más distante (ver Figura 2). Un análisis cuidadoso de la distorsión presente en este tipo de imágenes permite estimar la cantidad de masa que está en el camino y que está produciendo el lente gravitacional. Este tipo de estudios han mostrado que en el caso de cúmulos y de supercúmulos de galaxias, la cantidad de materia oscura es de 10 a 100 veces mayor que la cantidad de materia visible $[18,19]$. Actualmente, la cantidad y la calidad de los datos recolectados permiten hacer detallados mapas de la distribución de materia oscura en el universo observable. 


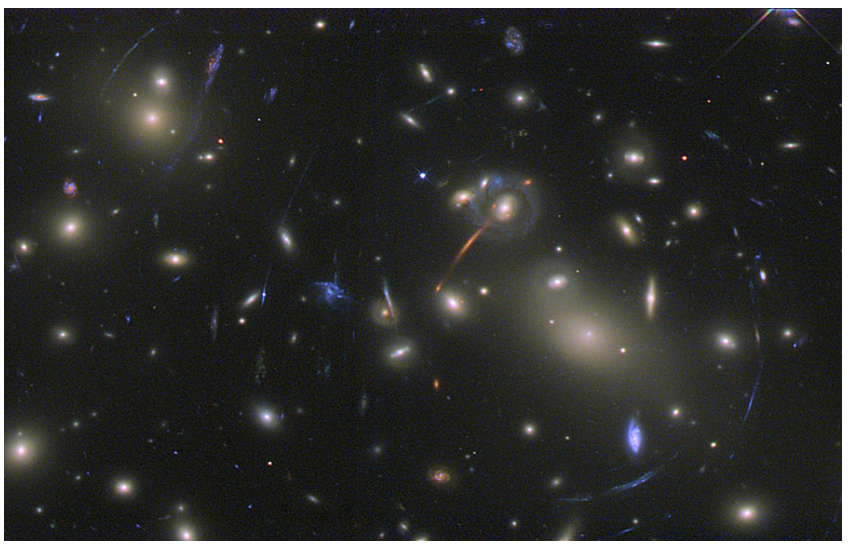

Figura 2: Imagen del lente gravitacional generado por el cúmulo Abell 2218. Los filamentos y las figuras arqueadas corresponden a galaxias más lejanas, cuyas imágenes son distorsionadas por el lente gravitacional generado por la masa del cúmulo que, en su gran mayoría, no es visible (imagen tomada de [20]).

Por último, el estudio de la formación de galaxias a partir del Big-Bang, a la luz de la información de alta precisión provista por el espectro de radiación cósmica de fondo, parece indicar que son necesarias grandes cantidades de materia oscura que aporten el campo gravitacional necesario para explicar este proceso [21].

En resumen, la evidencia a favor de la existencia de materia oscura en el universo es enorme, aún cuando hay algunos resultados contradictorios. La curvas de rotación de galaxias espirales, la dinámica de los cúmulos galácticos y el estudio de las imágenes de lente gravitacional, proveen argumentos muy convincentes que indican una cantidad de materia oscura en el universo que es unas 10 veces mayor que la cantidad de materia visible.

¿Cuál es la naturaleza de esta materia oscura? ¿Podría ser materia ordinaria que, por algún motivo, no es visible o detectable, o más bien, se trata de una nueva forma de materia? ¿Puede alguno de los candidatos provenientes de modelos BSM ser el constituyente microscópico de esta nueva forma de materia?

\section{COMPOSICIÓN DE LA MATERIA OSCURA}

Si en el universo hay grandes cantidades de materia que no vemos, ¿podría esta materia ser simplemente materia ordinaria, de carácter bariónico, que no emite luz, o que emite muy poca? Los halos galácticos podrían estar compuestos de objetos macroscópicos, suficientemente pequeños como para no ser detectados. Nada excluye la posibilidad de que existan cuerpos del tamaño de rocas o de asteroides flotando alrededor de las galaxias. Pero lo que sí es muy difícil de justificar es el tipo de procesos que habrían llevado a que la gran mayoría de la materia en el universo hubiese terminado de esta forma. Cuerpos de este tamaño están compuestos por elementos químicos pesados (tan pesados como el carbono, o más) y estos elementos solo son producidos como resultado de explosiones de supernovas, lo cual los hace muy poco abundantes. Sería más probable que hubiese una gran cantidad de objetos más grandes, pero de baja luminosidad, como por ejemplo enanas blancas, estrellas de neutrones o agujeros negros. Estos cuerpos constituyen el remanente de estrellas muertas, compuestas originalmente por hidrógeno y por helio. Las estrellas de neutrones y los agujeros negros son resultado de la explosión de estrellas muy masivas, que son poco abundantes. Existe la posibilidad de que en etapas tempranas del universo se hubiesen creado grandes cantidades de estrellas muy masivas, que, rápidamente, habrían colapsado en forma de agujeros negros. Otra posibilidad son las enanas rojas, estrellas muy pequeñas, que, debido a su baja luminosidad, son muy difíciles de observar. También podrían ser enanas cafés, cuerpos unas decenas de veces más grandes que Júpiter, que no alcanzan a convertirse en estrellas, y que, por tanto, emiten muy poca radiación. Si bien las enanas rojas o cafés son muy difíciles de detectar, los nuevos instrumentos astronómicos de alta resolución han permitido observar una cantidad suficiente de ellas, como para hacernos una idea de su abundancia. Nuevamente, la cantidad de este tipo de objetos no es suficiente para explicar la composición de la materia oscura, o al menos, de una fracción significativa de ella.

Si bien muchos de estos candidatos no son del todo plausibles, no pueden ser descartados. Varios equipos de investigadores han llevado a cabo búsquedas de objetos masivos y compactos en el halo galáctico (MACHO, MAssive Compact Halo Object). La forma de detectar estos cuerpos, tan pequeños y oscuros, es usando la técnica de Microlente Gravitacional (microlensing), debido a que, si bien, el campo gravitacional de un MACHO es muy pequeño, alcanza a desviar la luz que pasa cerca de su superficie. Cuando uno de estos MACHOs se atraviesa en la línea de visión de una estrella que está siendo observada desde la Tierra, el pequeño lente gravitacional que produce va a enfocar una mayor cantidad de luz sobre el observador (tal y como lo hace una lupa). El resultado es que durante el período en el cual el MACHO está atravesando la línea de visión, se observará un aumento en el brillo aparente de la estrella, produciendo una curva de luminosidad característica. El brillo de la estrella puede aumentar en unas cuantas decenas y la duración del pico de luminosidad puede ser de unos cuantos días.

Los lugares más adecuados para buscar eventos de microlente gravitacional son la Gran Nube de Magallanes y la Pequeña Nube de Magallanes, galaxias satélites de la Vía Láctea. Dada la cercanía de estas galaxias, podemos 
distinguir estrellas en forma individual. Cuando un MACHO perteneciente a la Vía Láctea se interpone en la línea de visión de una estrella en una de las nubes de Magallanes, el fenómeno del microlente se observa desde la Tierra. Desde la década de 1990, varios equipos de astrónomos han monitoreado una gran cantidad de estrellas en las nubes de Magallanes, en búsqueda de éste tipo de eventos $[22,23]$. Dada la dificultad de estas búsquedas, los resultados no han sido concluyentes, pero la evidencia indica que la contribución de los MACHOs a la masa del halo galáctico no puede ser mayor al $20 \%$, y, muy probablemente, no supera el $10 \%$. Si bien los MACHOs existen, no son la respuesta al problema de la materia oscura.

Dado que la información que poseemos sobre la materia oscura proviene en gran medida de sus efectos gravitacionales, bien podría ser que esta materia no exista, sino que sea el resultado de efectos gravitacionales a gran escala, cuya naturaleza ignoramos. Esta es la idea básica de los modelos teóricos de Dinámica Newtoniana Modificada (MOND, MOdified Newtonian Dynamics). En la versión original de éste tipo de teorías, presentada por Mordehai Milgrom en 1983 [24-26], el comportamiento de la fuerza gravitacional depende de la aceleración del cuerpo. Si esta aceleración es mayor que un valor de umbral, la fuerza gravitacional se comporta de la manera Newtoniana, es decir, inversamente proporcional al cuadrado de la distancia entre los cuerpos. Sin embargo, si la aceleración es menor que este valor, la fuerza se vuelve inversamente proporcional a la distancia. Para estrellas ubicadas en el disco de galaxias espirales, la aceleración es tan pequeña que la fuerza gravitacional, según MOND, sería inversamente proporcional a la distancia al centro galáctico, resultando en velocidades de rotación constantes y explicando las observaciones astronómicas para este tipo de sistemas. Otras teorías, basadas en cambios en el valor de la constante gravitacional de Newton, debido al carácter cuántico de campo gravitacional, también pueden reproducir muchos de los resultados de dinámica gravitacional a nivel galáctico e incluso cosmológico [27].

Si bien las teorías tipo MOND explican la dinámica galáctica, tienen problemas para explicar objetos como VIRGOHI21, un cuerpo muy masivo, pero que posee cantidades muy pequeñas de masa luminosa. De igual forma, MOND tiene problemas para explicar la gran cantidad de información, muy detallada, proveniente de las imágenes de lente gravitacional. Recientemente, una nueva evidencia a favor de la existencia de la materia oscura, y contraria a soluciones del tipo MOND, ha surgido a partir del estudio del cúmulo Bala, en la constelación de Carina. En realidad, se trata de dos cúmulos de galaxias, alejándose el uno del otro después de haber colisionado. Los dos cúmulos están compuestos por materia bariónica, en forma de galaxias y de gas, y por materia que solo se manifiesta gravitacionalmente, es decir, materia oscura. La materia en forma de galaxias ocupa un volumen muy pequeño, mientras que el gas se esparce por todo el volumen de los dos cúmulos. Como resultado de la colisión, las galaxias solo sufrieron un retardo en su movimiento debido a la interacción gravitacional. Por el contrario, la interacción electromagnética entre los gases de los dos cúmulos deformó sus distribuciones espaciales, como lo muestran las imágenes de rayos X. En el caso de la materia oscura, su presencia ha sido inferida a partir del estudio de imágenes de lente gravitacional. Si la materia oscura es no interactuante (collisionless) o, más bien, solo interactúa débilmente, las nubes de los dos cúmulos debieron haber pasado, la una a través de la otra, sin deformarse. Si asumiéramos MOND, la deformación de las nubes de gas causaría una deformación similar en el campo gravitacional y, por tanto, en las imágenes de lente gravitacional. Los resultados de los estudios de rayos $\mathrm{X}$ y de lente gravitacional para el cúmulo Bala muestran que las nubes de materia bariónica sufrieron la deformación esperada, mientras que las nubes de materia oscura, no (ver Figura 3) [28]. La evidencia muestra la existencia de un tipo de materia con efectos gravitacionales notorios, pero que no participa en colisiones. Es decir, materia que no interactúa ni electromagnética ni fuertemente.

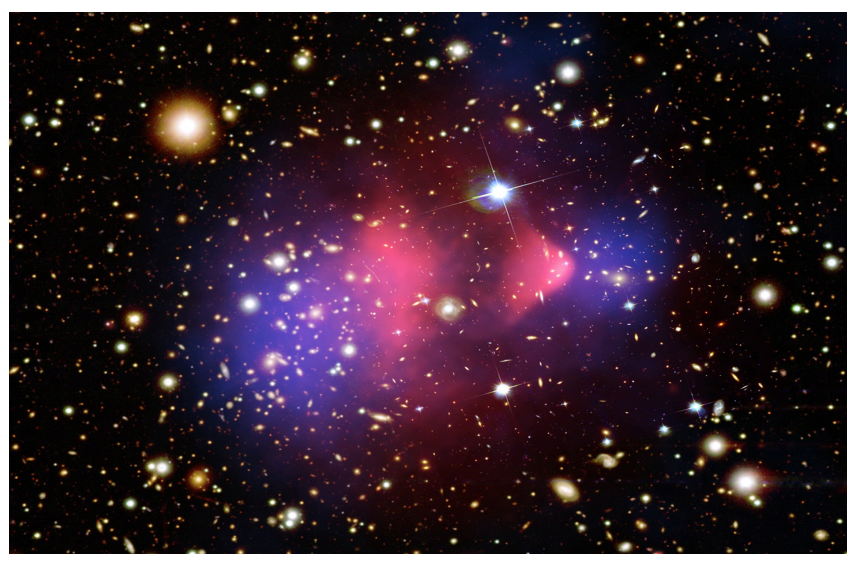

Figura 3: Imagen compuesta del cúmulo Bala. El cúmulo pequeño, del lado derecho de la imagen, atravesó al cúmulo grande. El color rojo representa la distribución de materia bariónica en forma de galaxias y de gas. El color azul representa la distribución de materia oscura. Como resultado de la colisión, la materia bariónica sufrió una deformación y un retardo, mientras que la materia oscura no sufrió ninguna deformación (imagen tomada de [29]).

Otro resultado importante a favor de la existencia de materia oscura no-bariónica emerge del proceso de nucleosíntesis durante el Big-Bang. Estudios de este proceso indican que, dada la abundancia de hidrógeno, helio, deuterio y litio en el universo, la densidad de materia bariónica necesaria para generarlas debería ser menor al $10 \%$ de la densidad de energía total [30-32]. 
Si se evalúan todos los resultados astronómicos en perspectiva, se puede concluir que hay fuertes evidencias a favor de la existencia de una materia oscura que está compuesta por partículas muy masivas, que no son bariones, que no poseen carga eléctrica y que, a lo sumo, interactúan débilmente.

\section{ESTRUCTURA MICROSCÓPICA DE LA MATERIA OSCURA}

Múltiples modelos teóricos proponen candidatos para constituir la materia oscura a nivel microscópico. Todos estos modelos deben representar, de una forma $u$ otra, extensiones del SM para poder explicar la naturaleza no-bariónica de la materia oscura y, a la vez, no contradecir a la física de partículas ya establecida. La solución más sencilla habría sido uponer que la materia oscura estuviese compuesta por partículas conocidas, pertenecientes al SM, como por ejemplo, los neutrinos. Pero la masa de estas partículas es tan pequeña que tendría que haber una cantidad enorme de ellas deambulando por el universo, en contradicción con toda la evidencia astronómica y con los modelos cosmológicos de evolución a partir del Big-Bang [33, 34]. Otra posibilidad es que existieran neutrinos con helicidad derecha, que tuvieran alta masa (la helicidad hace referencia a la orientación del espín de la partícula con respecto a su dirección de movimiento). Sin embargo, los neutrinos del SM solo interactúan débilmente y poseen helicidad izquierda. $\mathrm{Al}$ ser los neutrinos partículas masivas, podrían tener helicidad derecha pero, entonces, no interactuarían débilmente $\mathrm{y}$, dado que no poseen carga eléctrica ni tampoco carga de color, solo interactuarían gravitacionalmente. A este tipo de neutrinos se los denomina estériles. En los modelos teóricos en los que se incluyen neutrinos estériles, la masa de estas partículas puede llegar a tener valores muy altos, convirtiéndolas en candidatos a ser constituyentes de la materia oscura [35].

Otra posibilidad es que el espacio-tiempo en el que vivimos tenga más de 4 dimensiones. Además de la dimensión temporal y de las 3 dimensiones espaciales, podrían haber dimensiones espaciales extra, compactificadas hasta escalas microscópicas, de tal forma que no las notaríamos a nivel macroscópico. Excitaciones de Kaluza-Klein (KK) de los campos/partículas del SM en un universo con dimensiones espaciales extra (ED), le darían mayor masa a estos campos/partículas. Modelos teóricos con ED proponen excitaciones de KK como candidatos a ser constituyentes de la materia oscura [36, 37].

Los candidatos más populares a ser los constituyentes de la materia oscura provienen de modelos SUSY. Todos estos modelos recurren a una extensión de la simetría espacio-temporal, denominada supersimetría, que coloca a los campos fermiónicos y bosónicos del SM en un mis- mo plano, juntándolos en supercampos cuyas partículas son las que conocemos en el SM, sumadas a compañeras supersimétricas de mayor masa. Diferentes versiones de este tipo de teorías incluyen candidatos a constituir la materia oscura, como por ejemplo, los neutralinos en el caso de modelos que conservan la paridad $R$, o los gravitinos en el caso de modelos con rompimiento de SUSY mediado por gauge, o los s-neutrinos en algunas otras parametrizaciones.

Otros candidatos más exóticos han sido considerados; como por ejemplo: axiones [38], materia oscura ligera [39], materia oscura superpesada [40], partículas provenientes de modelos con Little Higgs [41], Q-balls [42], partículas espejo [43], partículas cargadas masivas (CHAMP, CHarged Massive Particle) [44], materia D (D-matter) [45], materia oscura en forma de branas [46], cryptons [47], entre otros. Si bien algunos de estos candidatos son menos plausibles, no pueden ser descartados y las diferentes búsquedas de materia oscura deben tener en cuenta las señales que ellos producirían. En este artículo nos concentraremos en los candidatos supersimétricos.

\section{MATERIA OSCURA SUPERSIMÉTRICA}

Como ya se ha mencionado anteriormente, existen varios argumentos teóricos para sospechar que el SM es una teoría efectiva para energías inferiores a los TeVs. Además de sus 28 o más parámetros libres, y de no proporcionar una explicación microscópica para la materia oscura ni para la energía oscura, a nivel de teoría cuántica de campos, el SM exhibe problemas que hacen poco satisfactoria su extensión a escalas de energía superiores. Entre estos problemas está el denominado falta de naturalidad de la masa del Higgs.

El problema de la falta de naturalidad de la masa $m_{H}$ del campo de Higgs emerge cuando se calculan correcciones radiativas a su propagador. En la Figura 4 se muestra la contribución al orden más bajo, debido a loops fermiónicos.

La contribución a la función de dos puntos, asociada a este tipo de diagrama, es [48]:

$\Pi_{H H}^{f}(0)=-2 \lambda_{f}^{2} \int \frac{d^{4} k}{(2 \pi)^{4}}\left[\frac{1}{k^{2}-m_{f}^{2}}+\frac{2 m_{f}^{2}}{\left(k^{2}-m_{f}^{2}\right)^{2}}\right]$,

donde $\lambda_{f}$ es la constante de acoplamiento entre el campo fermiónico y el campo de Higgs y $m_{f}$ es la masa del fermión. Esta integral es cuadráticamente divergente e independiente de $m_{H}$. Si el límite superior de la integral se corta a un valor de momentum $\Lambda$, del orden de la masa de Planck, entonces la corrección $\delta m_{H}^{2}$ es de unos 30 ordenes mayor que $m_{H}^{2}$ mismo. Además, si se lleva $m_{H}$ a cero, la corrección no se hace cero. Esto indica que no existe una simetría en el SM que proteja la masa 


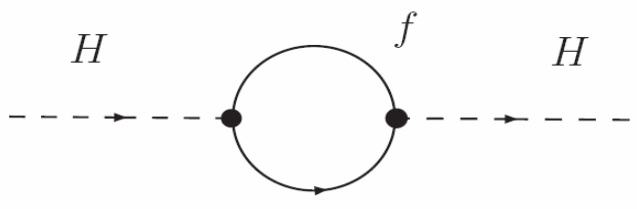

Figura 4: Corrección al propagador del campo de Higgs, $H$, debido a un loop fermiónico, $f$.

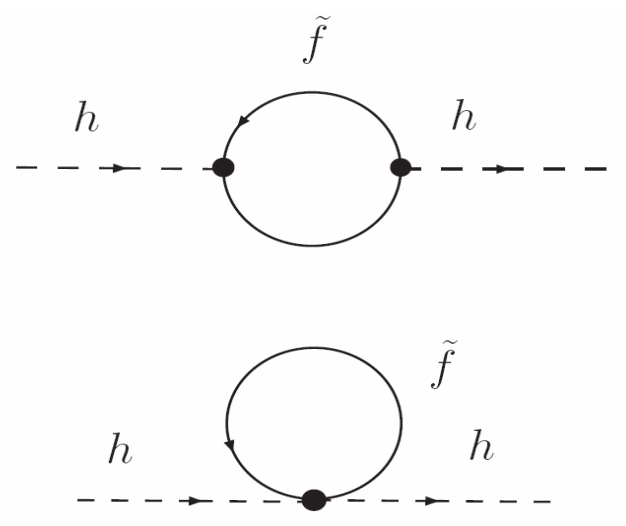

Figura 5: Corrección debida a loops s-fermiónicos al propagador de $h$.

del Higgs, haciendo de este un parámetro no-natural. Aún cuando esta divergencia se puede renormalizar de la forma habitual, en una extensión de la teoría a la escala de gran-unificación $\left(\sim 10^{16} \mathrm{GeV}\right)$, la renormalización de la masa del Higgs requeriría una cancelación de términos con una precisión de $\sim 10^{-26}$, lo cual es considerado poco natural e insatisfactorio [48].

La supersimetría es una extensión del grupo de Poincaré, que es el grupo de simetría fundamental del espacio-tiempo a un grupo de Super-Poincaré que, además de continuar siendo un grupo de simetría del espacio-tiempo, transforma campos bosónicos en fermiónicos y viceversa [49]. Esta supersimetría permite construir teorías de campos en términos de supermul- tipletes de partículas de igual masa que incluyen igual número de grados de libertad fermiónicos que bosónicos. Regresando al problema de la falta de naturalidad de $m_{H}$ en una extensión SUSY del SM, por cada loop fermiónico habría que incluir un loop bosónico (s-fermiónico) con igual constante de acoplamiento, pero con signo opuesto, como se muestra en la Figura 5.

Con la inclusión de estos términos, las divergencias cuadráticas en el propagador del campo de Higgs desaparecen $[50,51]$. Las divergencias remanentes son logarítmicas y dependen de $m_{H}$ en forma tal que, cuando este parámetro se lleva a cero, estas desaparecen. La teoría recupera así su naturalidad y las extensiones a escalas de energía superiores se hacen viables. Esta es la razón fundamental por la cual, aún después de más de treinta años de búsqueda experimental fallida, SUSY continúa siendo una teoría deseable [52].

Un modelo de teoría de campos tipo SUSY está escrito en términos de supermultipletes de partículas de igual masa. Estos supermultipletes pueden ser:

- Supermultipletes quirales : $\left(\frac{1}{2}, 0\right) \rightarrow(f, \tilde{f})$

- Supermultipletes de gauge $:\left(1, \frac{1}{2}\right) \rightarrow(g, \tilde{g})$

- Supermultiplete del gravitón : $\left(2, \frac{3}{2}\right) \rightarrow(G, \tilde{G})$.

A este nivel, el SM no puede reescribirse en términos supersimétricos, ya que los fermiones y bosones conocidos no se pueden poner juntos en supermultipletes, debido a que sus números cuánticos no lo permiten. La única forma de implementar una teoría de este tipo para las partículas elementales conocidas, es introduciendo nuevas compañeras SUSY de igual masa para cada una de las partículas del SM. Dado que ninguna de las partículas SUSY ha sido observada experimentalmente en el rango de masas conocido, SUSY debe ser una simetría rota. La ruptura de esta simetría debe ser tal que genere una diferencia de masas entre las partículas del SM y sus compañeras SUSY, del orden de cientos de $\mathrm{GeV}$, hasta varios $\mathrm{TeV}$, para no entrar en contradicción con las observaciones experimentales.

La versión más económica de SUSY, que incluye en forma adecuada al SM, es el Modelo Mínimo Supersimétrico Estándar (MSSM, Minimal Supersymmetric Standard Model). Este modelo posee el siguiente espectro de partículas: 


\section{SM}

$$
\begin{aligned}
& \text { leptones cargados } \rightarrow l^{ \pm} \\
& \text {neutrinos } \rightarrow \nu_{l} \\
& \text { quarks } \rightarrow q \\
& \text { bosones vectoriales cargados } \rightarrow W^{ \pm} \\
& \text {bosones vectoriales neutros } \rightarrow \gamma, Z^{o} \\
& \text { gluones } \rightarrow g \\
& \text { higgs } \rightarrow H
\end{aligned}
$$

\section{MSSM}

$$
\begin{aligned}
& \text { s-leptones } \rightarrow \tilde{l}^{ \pm} \\
& \text {s-neutrinos } \rightarrow \tilde{\nu}_{l} \\
& \text { s-quarks } \rightarrow \tilde{q} \\
& \text { Winos } \rightarrow \tilde{W}^{ \pm} \\
& \text {fotino, Zino } \rightarrow \tilde{\gamma}, \tilde{Z} \\
& \text { gluinos } \rightarrow \tilde{g} \\
& \text { higgsinos } \rightarrow \tilde{H}_{1}^{o}, \tilde{H}_{2}^{o}
\end{aligned}
$$

El rompimiento de la simetría electrodébil presente en el SM, se manifiesta en el sector supersimétrico en términos de una mezcla entre los gauginos (Winos, Binos, y higgsinos), resultando en un nuevo conjunto de estados observables denominados charginos y neutralinos, y un nuevo conjunto de campos de Higgs, como se muestra a continuación:

$$
\begin{aligned}
& \text { charginos ligeros } \rightarrow \tilde{\chi}_{1}^{ \pm} \\
& \text {charginos pesados } \rightarrow \tilde{\chi}_{2}^{ \pm} \\
& \text {neutralinos } \rightarrow\left\{\begin{array}{l}
\tilde{\chi}_{1}^{o} \leftarrow \text { el más ligero } \\
\tilde{\chi}_{2}^{o} \\
\tilde{\chi}_{3}^{o} \\
\tilde{\chi}_{4}^{o} \leftarrow \text { el más pesado }
\end{array}\right. \\
& \text { higgs } \rightarrow\left\{\begin{array}{l}
h \leftarrow \text { el más ligero } \\
H \\
A \\
H^{ \pm}
\end{array}\right.
\end{aligned}
$$

El modelo MSSM incluye un número cuántico multiplicativo que se conserva denominado Paridad $R$. La conservación de esta cantidad fue introducida inicialmente en la teoría para prevenir el decaimiento del protón. La Paridad $R$ está definida como:

$$
R \equiv(-1)^{3 B+L+2 S},
$$

donde $B$ es el número bariónico, $L$ es el número leptónico y $S$, el espín. De esta definición se puede deducir que $R=+1$ para todas las partículas del SM y $R=-1$ para todas las compañeras SUSY (s-partículas).
La conservación de la paridad $R$ tiene las siguientes consecuencias:

- Cualquier decaimiento de una s-partícula contiene otra s-partícula.

- La partícula SUSY más ligera (LSP, Lightest Supersymmetric Particle) tiene que ser estable.

- Toda la materia SUSY termina decayendo en LSP.

- En una colisión como las del LHC, las s-partículas tienen que ser creadas en pares.

- En una colisión como las del LHC, tiene que haber al menos un par de LSP en el estado final.

En el modelo MSSM, la LSP es $\tilde{\chi}_{1}^{o}$ que solo interactúa débilmente y es estable. Esta partícula se comporta en forma similar a un neutrino, solo que a diferencia de este, es muy masiva.

Las Figuras 6 y 7 muestran los canales de decaimiento de charginos y neutralinos. En el Cuadro I se listan los canales de decaimiento más importantes de s-leptones, de s-quarks y de gluinos. Como se puede constatar a partir de esta información, todas las partículas SUSY generan cadenas de decaimientos que llevan a charginos y a neutralinos, hasta que finalmente se llega a los neutralinos más ligeros, $\tilde{\chi}_{1}^{o}$, que son las LSP del MSSM. Como consecuencia de todo esto, si en las etapas tempranas del universo se produjeron grandes cantidades de materia SUSY, toda ella debió decaer rápidamente en partículas del SM y neutralinos $\tilde{\chi}_{1}^{o}$. Estos neutralinos serían los constituyentes microscópicos de la materia oscura que observamos. Por otro lado, en una colisión entre dos protones a una energía suficientemente alta, como lo son las colisiones del LHC, se podría producir materia SUSY, que generaría una cadena de decaimientos, terminando en un par de $\tilde{\chi}_{1}^{o}$. Estas partículas no dejan trazas en los detectores, pero transportan una cantidad apreciable de momentum 

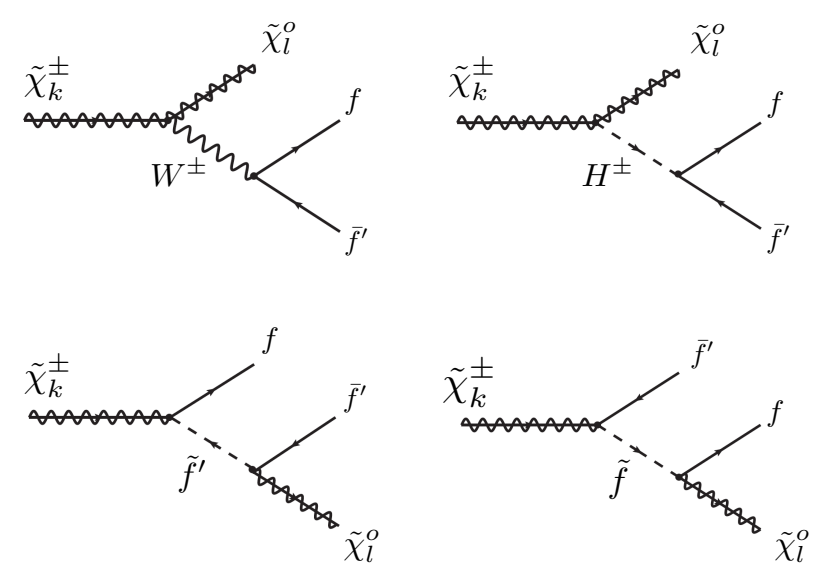

Figura 6: Decaimientos de Charginos según el MSSM [48].
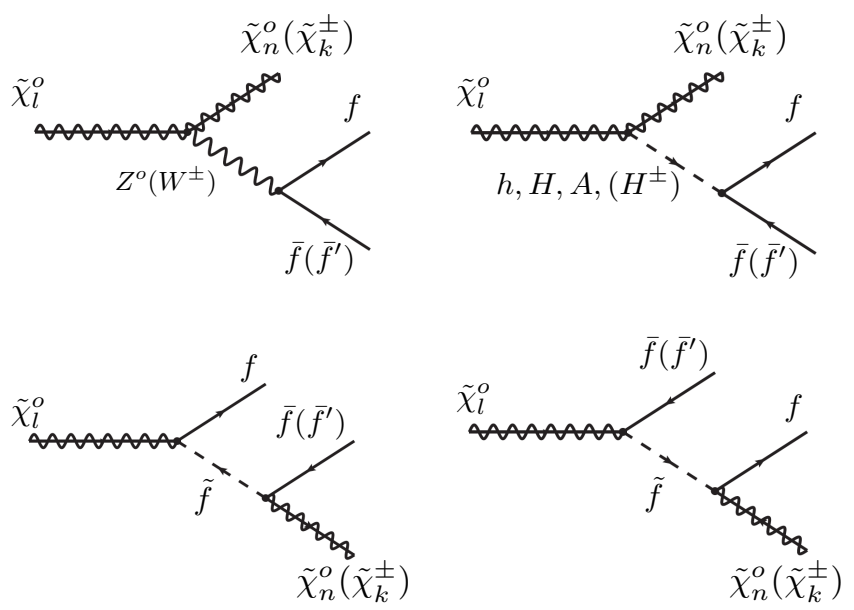

Figura 7: Decaimientos de Neutralinos según el MSSM [48].

y de energía; es decir, dejarían faltantes de energía y momentum significativos. La observación de colisiones de alto momentum transversal con cantidades igualmente altas de energía transversal faltante, consistente con predicciones de SUSY, representaría el descubrimiento de neutralinos y, por tanto, la observación indirecta de materia oscura de naturaleza supersimétrica.

En el SM, los campos de gauge $W^{ \pm}, Z^{o}$ y $\gamma$, son resultado de la mezcla de los campos $W_{1}, W_{2}, W_{3}$ y $B$. Los compañeros SUSY de los campos de gauge se pueden expresar en una base o en la otra. Por ejemplo, los 4 neutralinos del MSSM son resultado de la mezcla de los gauginos $\tilde{B}$ y $\tilde{W}_{3}$, y de los higgsinos $\tilde{H}_{1}^{0}$ y $\tilde{H}_{2}^{0}$. El neutralino más ligero, $\tilde{\chi}_{1}^{o}$, se puede escribir como [53]:

$$
\tilde{\chi}_{1}^{o}=N_{11} \tilde{B}+N_{12} \tilde{W}_{3}+N_{13} \tilde{H}_{1}^{0}+N_{14} \tilde{H}_{2}^{0}
$$

\begin{tabular}{|c|c|}
\hline s-partículas & Decaimientos \\
\hline s-leptones : & \\
\hline$\tilde{l}^{ \pm}$ & $\begin{array}{l}\rightarrow l^{ \pm} \tilde{\chi}_{k}^{o} \\
\rightarrow \nu_{l} \tilde{\chi}_{k}^{ \pm}\end{array}$ \\
\hline$\tilde{\nu}_{l}$ & $\begin{array}{l}\rightarrow \nu_{l} \tilde{\chi}_{k}^{o} \\
\rightarrow l^{ \pm} \tilde{\chi}_{k}^{\mp}\end{array}$ \\
\hline gluinos y s-quarks & \\
\hline Si $m_{\tilde{q}}<M_{\tilde{g}}$ & \\
\hline$\tilde{g}$ & $\rightarrow q \tilde{q}$ \\
\hline$\tilde{q}$ & $\begin{array}{l}\rightarrow q \tilde{\chi}_{l}^{o} \\
\rightarrow q^{\prime} \tilde{\chi}_{k}^{ \pm} \\
\rightarrow q \bar{b} \tilde{b}+q b \overline{\tilde{b}}\end{array}$ \\
\hline Si $m_{\tilde{q}}>M_{\tilde{g}}$ & \\
\hline$\tilde{g}$ & $\begin{array}{l}\rightarrow q \bar{q} \tilde{\chi}_{l}^{o} \\
\rightarrow q \bar{q}^{\prime} \tilde{\chi}_{k}^{ \pm} \\
\rightarrow q \bar{q}^{\prime} \tilde{\chi}_{k}^{ \pm} \\
\rightarrow \bar{t} \tilde{t}+t \overline{\tilde{t}} \\
\rightarrow \bar{b} \tilde{b}+b \overline{\tilde{b}}\end{array}$ \\
\hline$\tilde{q}$ & $\begin{array}{l}\rightarrow q \tilde{g} \\
\rightarrow q \tilde{\chi}_{l}^{o} \\
\rightarrow q^{\prime} \tilde{\chi}_{k}^{ \pm}\end{array}$ \\
\hline
\end{tabular}

Cuadro I: Decaimientos de s-leptones, de s-quarks y de gluinos según el MSSM [48].

donde $N_{i j}$ son elementos de la matriz de mezcla para los neutralinos.

A pesar de su simplicidad, el MSSM posee un número enorme de parámetros libres, del orden de 100, que permiten un amplio espectro de posibilidades teóricas. Para reducir este número de posibilidades de tal forma que se puedan hacer predicciones concretas que faciliten las búsquedas experimentales, es necesario hacer algunas suposiciones que tengan un sustento teórico sólido. Por ejemplo, cuando en un modelo SUSY promovemos la supersimetría a un nivel local, en forma similar a como construimos campos gauge en el SM, debemos incluir un super-campo gauge, que resulta siendo el campo gravitacional, mediado por el gravitón (una partícula sin masa de espín 2) y su compañero SUSY, el gravitino (masivo y de espín 3/2). Esta versión de SUSY es conocida como Supergravedad (SUGRA, SUper GRAvity) [54]. En este escenario el número de parámetros libres se reduce asumiendo ciertas condiciones de frontera a la escala de Gran Unificación. 
Otra posibilidad para construir diferentes modelos SUSY es explorar el mecanismo de rompimiento de supersimetría. Existen múltiples posibilidades que resultan en diferentes versiones de SUSY. Por ejemplo, rompimiento de SUSY mediado por anomalías (AMSB, Anomaly Mediates Symmetry Breaking) [55, 56]; rompimiento de SUSY mediado por gravedad (GMSB, Gravity Mediated Symmetry Breaking) [57]; rompimiento de SUSY mediado por gauge [58-60]; rompimiento de SUSY mediado por gauginos [61, 62]; entre otros. Cada uno de estos escenarios predice diferentes tipos de LSP. Por ejemplo, en el caso de SUGRA, el neutralino $\tilde{\chi}_{1}^{o}$ continúa siendo la LSP, pero posee un alto contenido de higgsino; en el caso de AMSB, la composición del neutralino es dominada por el Wino; en el caso de rompimiento de SUSY mediado por gravedad o por gauge, el gravitino se convierte en la LSP.

En el caso de modelos SUSY en los cuales el neutralino $\tilde{\chi}_{1}^{o}$ es la LSP, la composición de este estado en términos de gauginos y higgsinos tiene consecuencias fenomenológicas con importantes implicaciones cosmológicas y experimentales [119]. Estudiar experimentalmente esta composición es crucial para poder inferir el modelo SUSY más viable, y para determinar las consecuencias que esto pueda tener al momento de describir la materia oscura.

\section{INTERACCIONES DE LA MATERIA OSCURA CON LA MATERIA ORDINARIA}

La existencia de partículas masivas, neutras y que, a lo sumo, interactúen débilmente, no garantiza que ellas sean las constituyentes primordiales de la materia oscura que observamos en el universo. Estas partículas, y las leyes a las que obedecen, deben ser tales que, de la mano con los modelos cosmológicos de expansión del universo, a partir de Big-Bang, se pueda explicar la abundancia actual de materia oscura. Supongamos una partícula $\chi$ con masa $m_{\chi}$, producida en forma copiosa durante los primeros instantes del Big-Bang. Si las condiciones son adecuadas, se puede llegar a establecer un equilibrio térmico que resulte en un valor estacionario para la abundancia de esta partícula. La densidad de equilibrio se alcanza cuando se igualan las tazas de creación y de aniquilación. La partícula $\chi$ y su antipartícula $\bar{\chi}$ pueden ser creadas en procesos del tipo $l \bar{l} \rightarrow \chi \bar{\chi}$, donde $l$ es alguna otra partícula. Este tipo de procesos se da, siempre y cuando, la temperatura del medio sea superior a $m_{\chi}$. De igual forma, también vamos a tener el proceso inverso: aniquilaciones del tipo $\chi \bar{\chi} \rightarrow l \bar{l}$. Las tazas de creación y de aniquilación van a tender a igualarse, llegando a un valor de equilibrio para la abundancia de $\chi$ que depende de la temperatura. En la medida en que la temperatura del universo disminuye, esta abundancia también disminuye. Cuando la temperatura cae por debajo de $m_{\chi}$ como resultado de la expansión del universo, el proceso de creación $l \bar{l} \rightarrow \chi \bar{\chi}$ es suprimido, dado que no se cuenta con suficiente energía cinética. El proceso de aniquilación $\chi \bar{\chi} \rightarrow l \bar{l}$ también es suprimido debido a la caída en la densidad de $\chi$ quedando una densidad remanente de $\chi$ (relic density). El valor de esta densidad va a depender de la naturaleza de la partícula $\chi$, de su masa y de los procesos de creación y de aniquilación involucrados. Por tanto, no basta con ser un WIMP; la física asociada al candidato debe ser la adecuada para reproducir la densidad remanente de materia oscura, si es que se quiere dar explicación a este problema.

Actualmente, los valores más exactos que se poseen de la densidad remanente de materia oscura y de materia bariónica provienen de ajustes numéricos de parámetros cosmológicos. Estos parámetros están restringidos por los datos de múltiples experimentos astronómicos, tales como la distribución espacial de galaxias en el Universo observable, el espectro de fluctuaciones de temperatura en el CMB y la abundancia de cúmulos de galaxias $[64,65]$ :

$$
\begin{aligned}
& \Omega_{D M} h^{2}=0,1198 \pm 0,0026 \\
& \Omega_{b} h^{2}=0,02207 \pm 0,00027
\end{aligned}
$$

donde $\Omega \equiv \rho / \rho_{\text {crit }}$ es el parámetro de densidad, $\rho_{\text {crit }}$ es la densidad crítica de masa del universo (que corresponde a un universo plano), $h$ es el parámetro adimensional de Hubble, y $\Omega_{D M}$ y $\Omega_{b}$ son los parámetros de densidad de la materia oscura y de la materia bariónica, respectivamente. Si la materia oscura está compuesta por WIMPs $\chi$, la densidad remanente de estas partículas estaría dada por [66]:

$$
\Omega_{\chi} h^{2}=\text { const. } \frac{T_{0}^{3}}{M_{\mathrm{PL}}^{3}\left\langle\sigma_{A} v\right\rangle} \simeq \frac{0,1 \mathrm{pb} \cdot c}{\left\langle\sigma_{A} v\right\rangle},
$$

donde $T_{0}$ es la temperatura actual de la radiación cósmica de fondo (CMB, Cosmic Microwave Background), $M_{\mathrm{PL}}$ es la masa de Planck, $c$ es la velocidad de la luz, $\sigma_{A}$ es la sección transversal total de aniquilación de $\chi, \mathrm{y}, v$ es la velocidad relativa entre los dos WIMPs, en el marco de referencia de centro de masa. Si la temperatura a la cual cesa la creación de $\chi$ es del orden de $T_{F} \simeq m_{\chi} / 20$ (freeze out temperature), la velocidad $v$ es no-relativista, lo cual, de acuerdo con la ecuación (8), resulta en $\sigma_{A}$, del orden de los pico-barns, es decir, del mismo orden de magnitud de las secciones transversales para interacciones débiles. Este tipo de resultados dan valor a la hipótesis de los WIMPs como constituyentes fundamentales de la materia oscura.

En el caso supersimétrico, como ya ha sido mencionado, en muchas de las parametrizaciones más plausibles, el WIMP es el neutralino más ligero, $\chi_{1}^{o}$. A pesar de no poseer carga eléctrica, ni carga de color, esta partícula 
sí interactúa débilmente, resultando en varios canales de aniquilación [67]:

$$
\begin{aligned}
& \text { - } \tilde{\chi}_{1}^{o} \tilde{\chi}_{1}^{o} \rightarrow f \bar{f} \\
& \text { - } \tilde{\chi}_{1}^{o} \tilde{\chi}_{1}^{o} \rightarrow \mathrm{W}^{+} \mathrm{W}^{-}, \\
& \text {- } \tilde{\chi}_{1}^{o} \tilde{\chi}_{1}^{o} \rightarrow \mathrm{Z}^{o} \mathrm{Z}^{o}, \\
& \text { - } \tilde{\chi}_{1}^{o} \tilde{\chi}_{1}^{o} \rightarrow g g \\
& \text { - } \tilde{\chi}_{1}^{o} \tilde{\chi}_{1}^{o} \rightarrow \gamma \gamma \\
& \text { - } \tilde{\chi}_{1}^{o} \tilde{\chi}_{1}^{o} \rightarrow \mathrm{W}^{ \pm}+\text {SUSY-Higgs, } \\
& \text { - } \tilde{\chi}_{1}^{o} \tilde{\chi}_{1}^{o} \rightarrow \mathrm{Z}^{o}+\text { SUSY-Higgs, } \\
& \text { - } \tilde{\chi}_{1}^{o} \tilde{\chi}_{1}^{o} \rightarrow \text { SUSY-Higgs, }
\end{aligned}
$$

donde SUSY-Higgs hace referencia a los campos de Higgs del MSSM: $\mathrm{h}^{o}, \mathrm{H}^{o}, \mathrm{~A}^{o}$ y $\mathrm{H}^{ \pm} ; f$ hace referencia a fermiones, $f=l, \nu_{l}, q$; donde $l=e, \mu, \tau ; \mathrm{y}$ $q=u, d, s, c, b, t$.

Si bien las secciones transversales de aniquilación de neutralinos son pequeñas, la cantidad de ellos en el cosmos sería enorme, de tal forma que habría una probabilidad finita de observar, a nivel astronómico, señales de estas aniquilaciones.

La materia oscura no solo interactúa con sí misma para aniquilarse, también puede interactuar con materia ordinaria, produciendo reacciones de dispersión elásticas e inelásticas (scattering) con núcleos atómicos. La interacción elástica de un WIMP con un núcleo puede causar que este retroceda con una energía del orden de decenas de $\mathrm{keV}$. La interacción inelástica puede generar una reacción nuclear que resulte en el retroceso y en la excitación del núcleo, seguido por la emisión de un fotón durante el proceso de desexcitación. Otra posibilidad es la interacción con un electrón atómico para producir su emisión, dejando al átomo en un estado ionizado. La probabilidad de estas interacciones en el caso supersimétrico depende de las secciones transversal neutralino-quark, neutralino-gluón y neutralino-electrón, que son calculables a partir de los modelos SUSY.

La producción de materia oscura supersimétrica durante las etapas tempranas del universo se debió haber dado por medio de las mismas reacciones de aniquilación mencionadas anteriormente pero en dirección opuesta y produciendo grandes cantidades de pares $\tilde{\chi}_{1}^{o} \tilde{\chi}_{1}^{o}$ o como resultado del decaimiento de materia supersimétrica más pesada. Actualmente la única forma de producir esta materia, con alguna esperanza de observarla, sería en las colisiones protón-protón del acelerador LHC, dada la alta energía de centro de masa requerida. En este tipo de colisiones, el proceso de producción sería: $p p \rightarrow \tilde{\chi}_{1}^{o} \tilde{\chi}_{1}^{o}+X$, donde $X$ representa un sinnúmero de posibles estados finales que involucran múltiples partículas y topologías cinemáticas. El par $\tilde{\chi}_{1}^{o} \tilde{\chi}_{1}^{o}$ producido escaparía a la detección, dado que solo interactúa débilmente, dejando un faltante de energía en los detectores. Este faltante de energía, sumado a una topología consistente para las demás partículas producidas e inconsistente con cualquier proceso conocido, representa la señal básica en la búsqueda de materia oscura por parte de los experimentos CMS y ATLAS del acelerador LHC.

\section{BÚSQUEDAS DIRECTAS DE MATERIA OSCURA}

La producción y detección de WIMPs por parte de los experimentos del LHC no necesariamente es prueba de que estas partículas sean las constituyentes básicas de la materia oscura. También es necesario observar señales de ellas a nivel astronómico, e incluso en la Tierra, ya que, supuestamente, están en todo lugar en la Vía Láctea. Múltiples experimentos buscan señales directas de estos WIMPs como resultado de colisiones con núcleos o electrones atómicos. De igual forma, otros experimentos buscan señales indirectas de la presencia de WIMPs en la Vía Láctea, al detectar partículas resultantes de su aniquilación.

Las técnicas experimentales que se usan en las búsquedas directas de WIMPs están inspiradas, en su gran mayoría, en los experimentos de detección de neutrinos [6870]. Los neutrinos, al igual que los WIMPs, solo interactúan débilmente, por tanto, los esquemas de detección y la sensibilidad requerida son similares. En el caso de la materia oscura, el orden de magnitud de la masa y de la sección transversal de interacción de un WIMP están, más o menos, determinados dependiendo del modelo teórico que provea el candidato. Al momento de estimar el número de eventos de colisión WIMP-Núcleo, la otra cantidad relevante es la densidad de materia oscura que deberíamos tener en la Tierra. Esta información proviene de los estudios astronómicos de la estructura del halo de materia oscura en la Vía Láctea, ya que nuestro planeta está sumergido en este halo. El perfil de densidad de materia oscura en galaxias espirales se puede extraer de los datos de velocidad de rotación de estrellas. Aun cuando la Vía Láctea es una galaxia espiral, su curva de velocidad de rotación no es tan bien conocida como la de otras galaxias cercanas (es más fácil medir las velocidades estelares de una galaxia haciéndolo desde afuera). Este tipo de estudios arrojan valores como: la velocidad del WIMP relativa al blanco: $\langle v\rangle \simeq 0,75 \times 10^{-3} c(220 \mathrm{~km} / \mathrm{s})$; la densidad local de materia oscura: $\rho_{0} \simeq 0,3 \mathrm{GeV} / \mathrm{cm}^{3}$; y la sección transversal WIMP-Núcleo: $\sigma_{W-N} \simeq 10^{-38}$ $\mathrm{cm}^{2}\left(10^{-2} \mathrm{pb}\right)$. Con estos valores, y una masa típica de un WIMP, $m_{\chi} \simeq 100 \mathrm{GeV} / \mathrm{c}^{2}$, podemos estimar, por ejemplo, la densidad numérica local de WIMPs, $n_{0}=\rho_{0} / m_{\chi} \simeq 3 \times 10^{-3}$ WIMPs $/ \mathrm{cm}^{3}$. El flujo de WIMPs en la Tierra sería $\phi_{0}=n_{0} \times\langle v\rangle \simeq 6,6 \times 10^{4} \mathrm{~cm}^{-2} s^{-1}$. 
El número de interacciones electro-débiles por unidad de tiempo y por unidad de masa del detector estaría dada por [71]:

$$
R \sim N_{N} \times \phi_{0} \times \sigma_{W-N} \sim 0,13 \text { eventos } \mathrm{kg}^{-1} \mathrm{año}^{-1},
$$

donde $N_{N}=N_{A} / A$ es el número de núcleos, $N_{A}$ es el número de Avogadro y $A$ es la masa atómica del material en el detector. La energía que cada una de estas colisiones le imprime a un núcleo es del orden de $30 \mathrm{keV}$ que es un valor muy pequeño en términos de detección. Para maximizar la energía transferida al núcleo, es deseable que $m_{\chi} \simeq m_{N}$; por tanto, se pueden usar varios blancos con diversos valores de $A$, y así poder restringir el valor de $m_{\chi}$ en caso de observar un evento. Dado el bajo número de eventos esperados por kilogramo de materia y por año, es necesario construir detectores con volúmenes muy grandes para maximizar el número de eventos registrados.

Debido a que la Tierra orbita alrededor del Sol y que este se mueve con respecto a la galaxia y a su halo, el flujo de WIMPs debería tener una dependencia estacional y también ser mayor en la dirección en la cual se mueve el Sol; esto es, en dirección a la constelación del Cisne. En un detector en el cual se pueda medir la dirección de los retrocesos de los núcleos, debería observarse un exceso de eventos con retrocesos en dirección opuesta a Cisne y también observarse variaciones estacionales. Por tanto, es deseable que el detector tenga esta capacidad, para así aumentar la certidumbre sobre el origen de los eventos registrados.

El principal reto que enfrentan los experimentos de búsquedas directas de materia oscura es entender y poner bajo control las señales de ruido en el detector (backgrounds); es decir, todos aquellos procesos físicos que puedan suceder en el detector o cerca de él, y que produzcan señales similares a las que se están buscando para los WIMPs. La principales fuentes de ruido en este tipo de experimentos son: la radioactividad en el aire debido a la presencia de radón; las impurezas de tipo radioactivo en el material de los detectores y del blindaje; los neutrones producto de la fisión nuclear y de otras reacciones nucleares que suceden en forma natural; los rayos cósmicos; y la activación de los materiales del detector debido a su exposición a la radioactividad en la superficie de la Tierra, antes de ser llevados a su lugar de funcionamiento.

Las fuentes de ruido asociadas a rayos cósmicos se pueden atenuar colocando el detector bajo tierra, lo más profundo que sea posible para proveer blindaje suficiente. Los hadrones presentes en el flujo de rayos cósmicos van a ser absorbidos al cabo de unos cuántos metros de profundidad y, por tanto, dejan de ser fuente de ruido. La situación es muy diferente con los muones que son partículas muy penetrantes. A grandes profundidades, solamente los muones más energéticos logran penetrar, pero son justamente estos los que pueden representar un mayor problema. Cuando un $\mu^{-}$ es capturado por un protón en un núclec

un neutrón y un neutrino, un proceso conocido como captura de muones negativos (negative muon capture), en el que los neutrones son emitidos con energías de decenas de GeV. Debido a interacciones con el medio, esta energía se va a atenuar hasta llegar al rango de los $\mathrm{MeV}$. La interacción de estos neutrones con núcleos en el volumen activo del detector, puede producir retrocesos con energías en el rango de los keV, que son las energías esperadas para los eventos asociados a WIMPs. Para controlar este ruido, es necesario rodear el detector central con detectores de veto, que alerten al sistema sobre la presencia de muones muy energéticos, o de cascadas de partículas, resultado de interacciones de estos muones con el medio. Otra fuente natural de neutrones y de ruido para estos experimentos es la presencia de partículas $\alpha$, emitidas como resultado de decaimientos radioactivos de ${ }^{238} \mathrm{U}$ y de ${ }^{232} \mathrm{Th}$, que están presentes en la roca que rodea al detector. Estas partículas pueden desencadenar reacciones nucleares que emitan uno o varios neutrones. Para controlar esta fuente de ruido, es necesario conocer en forma muy precisa la composición de todos los materiales y la cantidad de isotopos radioactivos presentes para así determinar, a partir de cálculos muy detallados, el flujo de neutrones producidos.

La principal fuente de ruido proviene de los neutrinos, ya que no existe ningún blindaje para este tipo de partículas. Los neutrinos provenientes del Sol, resultado de las reacciones del ciclo pp, pueden producir retrocesos de electrones atómicos con energías en el rango de los $\mathrm{keV}$; unos 20 eventos por tonelada y por año. La capacidad que tenga el detector para distinguir entre retrocesos electrónicos y nucleares es crucial para controlar esta señal.

Experimentos basados en detectores de estado sólido operados a temperaturas inferiores a $1 \mathrm{~K}$ son sensibles a energías menores a $10 \mathrm{keV}$ y tienen excelente resolución, pudiendo diferenciar entre retrocesos electrónicos y nucleares. Ejemplos de este tipo de experimentos son: CDMS, en el Laboratorio Subterráneo de Soudan en Minessota [72, 73]; CRESST, en el Laboratorio Nacional del Gran Sasso en Italia [74, 75] y EDELWEISS, en el Laboratorio Subterráneo de Modane en Francia [76, 77]. Experimentos con detectores de ionización de Germanio, operados a $77 \mathrm{~K}$, alcanzan energías de umbral de menos de un keV. Por ejemplo, CoGeNT, en el Laboratorio Subterráneo de Soudan [78], y TEXONO, en el Laboratorio de Neutrinos de Reactor de Kuo-Sheng en Taiwan [79]. Estos experimentos no pueden distinguir retrocesos electrónicos de retrocesos nucleares.

Experimentos basados en el uso de elementos nobles, en estado líquido como sustancia activa de detec- 
ción, ofrecen una alternativa al ser combinados con la tecnología de cámaras de proyección de tiempo (time-projection chambers). El uso de xenón líquido y de argón líquido, que pueden actuar como materiales centelladores al paso de partículas ionizantes, permiten la construcción de detectores de gran tamaño y de gran masa. Ejemplos de este tipo de experimentos son: ZEPLIN, en el Laboratorio Subterráneo de Boulby en el Reino Unido [80, 81]; XENON10 y XENON100, en el Laboratorio Nacional del Gran Sasso en Italia [75, 82]; XMASS, en Kamioka en Japón [83, 84]; y LUX, en Black Hills en Dakota del Sur [85].

Experimentos basados en el uso de cristales centelladores de ioduro de sodio (NaI) de alta pureza, ofrecen una alternativa con muy bajos niveles de ruido. Los experimentos del Proyecto DAMA [86], en el Laboratorio Nacional del Gran Sasso en Italia, en particular DAMA/LIBRA, son sensibles a variaciones estacionales en el flujo de WIMPs. Experimentos similares entrarán en funcionamiento muy pronto, como es el caso de ANAIS, en el Laboratorio Subterráneo de Canfrac en España [87, 88]; y DM-Ice, en la Antártica [89].

La sección transversal de interacción WIMP-Núcleo depende del espín del WIMP y del espín de núcleo. El espín del WIMP es desconocido. En el caso del neutralino más ligero de modelos SUSY, el espín es 1/2; pero, en otros modelos teóricos, puede tener un valor diferente. Los experimentos sensibles al espín del WIMP usan isotopos con espín impar, como el ${ }^{19} \mathrm{~F}$, en líquidos supercalentados. Ejemplos de este tipo de experimentos son: PICASSO, en SNOLAB en Canada [90, 91], que usa $\mathrm{C}_{4} \mathrm{~F}_{10}$; COUPP, en Fermilab [92], que usa $\mathrm{CF}_{3} \mathrm{I}$; y SIMPLE, en el Laboratorio Subterráneo de Bas Bruit en Francia [93, 94], que usa $\mathrm{C}_{2} \mathrm{ClF}_{5}$.

Las búsquedas directas de WIMPs no han arrojado resultados concluyentes hasta el momento. El experimento CoGeNT reportó evidencia de un WIMP con una masa de $7 \mathrm{GeV}$, pero esta partícula fue excluida por las búsquedas llevadas a cabo por CDMS, EDELWEISS y XENON10. El detector DAMA/LIBRA ha reportado evidencia de una dependencia estacional en el número de eventos de dispersión a bajas energías. Los experimentos DM-Ice y ANAIS que entrarán en servicio muy pronto, tienen como uno de sus objetivos principales verificar estos resultados. En los próximos años, nuevas colaboraciones pondrán en funcionamiento detectores más grandes y más sensibles, que permitirán explorar el espacio de parámetros de múltiples teorías en física de partículas que predicen candidatos a ser los constituyentes de la energía oscura.

\section{BÚSQUEDAS INDIRECTAS DE MATERIA OSCURA}

Otra opción en la búsqueda de materia oscura es la detección de señales de la aniquilación de WIMPs. Si bien se supone que los WIMPs son estables, esto no impide que al interactuar con ellos mismos se aniquilen, produciendo otras partículas como resultado de este proceso. Las partículas resultantes pertenecen al SM, y sabemos cómo detectarlas. La información cinemática que ellas entreguen nos proveerá información indirecta sobre las propiedades de los WIMPs, como, por ejemplo, su masa.

Aun cuando no conocemos las propiedades físicas de los WIMPs, dado que no los hemos observado, sí conocemos las propiedades de los candidatos más plausibles. En el caso de los neutralinos de SUSY, estas partículas son fermiones (tienen espín 1/2) y son sus propias antipartículas (partículas de Majorana). Por tanto, colisiones entre neutralinos podrían terminar en aniquilaciones. Dada la gran cantidad de materia oscura en el universo y, en particular en los halos galácticos, las colisiones entre neutralinos podrían suceder con suficiente frecuencia, a pesar de sus muy pequeñas secciones transversales de interacción, como para que sus señales sean detectables. Aun si los WIMPs no son sus propias antipartículas, todo indica que deberían haber cantidades similares de WIMPs y de anti-WIMPs en la materia oscura, de forma tal que deberían ocurrir aniquilaciones WIMP - anti-WIMP, con señales similares a las que producirían las aniquilaciones de neutralinos.

Las aniquilaciones de materia oscura deberían producir partículas estables como: fotones, electrones, positrones, neutrinos, protones y antiprotones; también podrían producirse partículas inestables, pero ellas decaerían rápidamente en las anteriores. Los protones y electrones se confundirían con la materia ordinaria del medio, pero fotones, neutrinos, positrones y antiprotones podrían producir señales distintivas. Las búsquedas indirectas de materia oscura se concentran en estas señales.

Las señales de aniquilaciones de materia oscura sucederían con muy poca frecuencia, al compararlas con señales similares producidas por procesos conocidos. Por tanto, se debe buscar primero en lugares donde sea mucho más probable que sucedan colisiones entre WIMPs. Al interior de una estrella o de un planeta, la probabilidad de que los WIMPs interactúen con núcleos atómicos perdiendo energía, y cayendo en el campo gravitacional del cuerpo, es más alta. Como resultado de este mecanismo, se esperarían mayores densidades de materia oscura al interior del Sol o de la Tierra que en el espacio interestelar y, en consecuencia, mayor probabilidad de colisiones entre WIMPs. Otro lugar con alta densidad de materia oscura sería el centro de la Vía Láctea. 
Las aniquilaciones de WIMPs atrapados en el centro de la Tierra producirían neutrinos muónicos. Algunos de estos neutrinos en camino hacia el exterior del planeta, podrían interactuar con núcleos del medio, produciendo muones, que tendrían una dirección de movimiento y una energía muy similares a las de los neutrinos de los cuales se originaron. Experimentos de neutrinos en la Tierra detectarían muones que vienen desde abajo. Un flujo de muones que viene desde arriba es normal en este tipo de detectores, debido a los rayos cósmicos; pero un flujo de muones que viene desde abajo indicaría la producción de neutrinos en el centro de la Tierra. Si la energía de los muones detectados, que es muy similar a la de los neutrinos originales, está en el rango de los $\mathrm{GeV}$ a los cientos de $\mathrm{GeV}$, sería consistente con aniquilaciones de materia oscura.

Búsquedas de este tipo de señales han sido llevadas a cabo por telescopios de neutrinos como: MACRO, en el Gran Sasso, en Italia [95]; BAKSAN, en Rusia [96]; SuperKamiokande, en Japón [97]; Lago Baikal, en Rusia [98]; AMANDA, en la Antártida [99]; ANTARES, en el mar Mediterráneo [100]; NESTOR, en el mar Egeo [101]; y IceCube, en la Antártida [102]. Hasta el momento, la estadística acumulada por estos experimentos no es suficiente como para obtener resultados concluyentes sobre la existencia de WIMPs en el centro de la Tierra. Algunos de estos experimentos pueden medir flujos de neutrinos provenientes del Sol, buscando señales con energías en el rango esperado para aniquilaciones de WIMPs. También, en este caso, los resultados aún no son concluyentes.

Las búsquedas astronómicas de señales de aniquilaciones de WIMPs se concentran en los fotones. Diversos canales de aniquilación resultan en cadenas de decaimientos que terminan emitiendo rayos gamma en un rango amplio de frecuencias. La cinemática de las partículas en el estado final del decaimiento, es tal que el espectro de gammas va a tener un valor máximo de energía (cut-off energy). Esta energía va a estar directamente relacionada con la masa de los WIMPs que se aniquilaron. La observación de cortes en el espectro de gammas provenientes de alguna región particular del espacio con valores de energía en la región de los cientos de $\mathrm{GeV}$, podría ser evidencia de estas aniquilaciones. En algunos canales de aniquilación, los dos WIMPs decaen directamente en dos fotones. En consecuencia, la energía de cada fotón va a ser igual a la masa del WIMP, salvo correcciones cinéticas. La señal de este tipo de eventos sería una línea espectral bien definida, con cierto ancho y con un valor central aproximadamente igual a la masa del WIMP. Desafortunadamente, muchos procesos astrofísicos que no tienen que ver con la presencia de materia oscura, emiten grandes cantidades de gammas en un amplio espectro, constituyéndose en ruido para este tipo de búsquedas.
Aniquilaciones de WIMPs en el halo de nuestra galaxia deberían producir un espectro continuo de gammas sumado a líneas espectrales provenientes de los canales de aniquilación a $\gamma \gamma$ y a $\gamma Z$. El tamaño de estas señales en alguna dirección particular está relacionado con la estructura del halo galáctico, que no es muy bien conocida. Sin embargo, lo que sí es claro es que estas señales deberían ser más fuertes en la dirección del centro de la galaxia. Desafortunadamente, el centro de la galaxia es una región muy activa, con muchas fuentes de rayos gamma de energías diversas. Experimentos como HESS, en Namibia [103]; y FERMI/LAT, en órbita [104], han observado fuentes puntuales de gammas con energías en los rangos de los $\mathrm{TeV}$ y de los $\mathrm{GeV}$, respectivamente. Sin embargo, la posibilidad de que estas fuentes tengan un origen astrofísico es muy alta. Un análisis independiente de los datos hechos públicos por FERMI/LAT, ha mostrado un exceso en el espectro alrededor de $\sim 130 \mathrm{GeV}$ [105]. Un nuevo análisis muy cuidadoso de este exceso, por parte de la Colaboración FERMI/LAT, ha reducido su significancia estadística, haciéndolo compatible con procesos astrofísicos conocidos.

Otras partículas que son producto de las aniquilaciones de WIMPs, y cuya señal puede sobresalir del ruido cósmico, son los anti-protones y los positrones. Aun cuando estas son partículas de anti-materia, son estables y, mientras no sean aniquiladas camino a la Tierra, podrían ser detectadas en órbita. Los antiprotones se producirían como resultado de la hadronización asociada a la producción de anti-quarks, en canales de aniquilación de WIMPs. Los positrones se producirían como resultado de canales con pares leptón - anti-leptón en el estado final. Al igual que en otras búsquedas, la señal producida por este tipo de procesos debería ser pequeña comparada con el ruido proveniente de otros procesos que producen antimateria a nivel cósmico. Por ejemplo, colisiones de rayos cósmicos con la materia ordinaria del medio interestelar producen importantes cantidades de antimateria en forma de positrones y de anti-protones. Observatorios en órbita como el satélite PAMELA y el detector AMS en la Estación Espacial Internacional, miden los flujos de positrones, anti-protones y anti-núcleos, y los comparan con los flujos de electrones, protones y núcleos en un amplio rango de energías. Excesos de partículas de antimateria con energías en el rango de las decenas de $\mathrm{GeV}$ podrían ser consistentes con aniquilaciones de materia oscura en el halo galáctico. AMS y PAMELA han reportado un incremento en la fracción de positrones detectados con energías entre 10 y $200 \mathrm{GeV}[108,109]$. Estos datos no pueden ser explicados fácilmente en términos de producción secundaria debida a rayos cósmicos, pero sí se podrían explicar como resultado de aniquilaciones de WIMPs. Sin embrago, las secciones transversales de aniquilación responsables de esta señal, serían demasiado altas para los modelos teóricos existentes, e inconsistentes con otros resultados. En resumen, las búsquedas 
indirectas de materia oscura han aportado algunos resultados intrigantes, pero estos aún son muy precarios e inconsistentes con otros resultados similares.

\section{BÚSQUEDAS DE MATERIA OSCURA EN EL LHC}

En cuanto a la búsqueda de materia oscura a nivel microscópico, la tercera opción es su producción y detección en aceleradores de partículas. Las pequeñas secciones transversales de interacción, y la alta masa que se espera para estas partículas, hace que su producción solo pueda darse en un colisionador de partículas de alta energía y de alta intensidad. Hoy en día, el único lugar donde se podría lograr esto es en el acelerador LHC del laboratorio CERN. El LHC es un acelerador circular que hace colisionar haces de protones que viajan en direcciones opuestas a altas energías. Los protones colisionan en 4 puntos diferentes a lo largo del anillo de $27 \mathrm{~km}$ de circunferencia del acelerador. Los 4 puntos de colisión están rodeados por 4 grandes detectores de partículas: ATLAS [110], ALICE [111], CMS [112] y LHCb [113]. Dos de ellos, muy similares en su diseño $\mathrm{y}$ en sus objetivos científicos, ATLAS y CMS, son experimentos optimizados para la búsqueda de nueva física, más allá del Modelo Estándar. Su primer objetivo científico ya fue alcanzado en gran medida: el descubrimiento y el estudio del bosón de Higgs. Su segundo objetivo científico, la búsqueda de física BSM aún no ha arrojado resultados positivos. Entre los modelos teóricos candidatos a ser los responsables por esta nueva física, los más estudiados y más elaborados son los modelos SUSY. Si bien el objetivo fundamental de ATLAS y de CMS es la búsqueda de nueva física, es muy probable que cualquier descubrimiento en esta dirección venga de la mano con respuestas al problema de la materia oscura. Hemos mencionado el caso de algunos modelos SUSY que proveen un candidato para ser el muy ansiado WIMP: el neutralino más ligero. El descubrimiento de señales experimentales de producción de materia SUSY con un par de neutralinos en el estado final, podría significar un enorme paso en la lucha por entender la estructura microscópica de la materia oscura. Como ya ha sido mencionado, no solo se trata de observar estos neutralinos; las masas y las secciones transversales deben ser consistentes con los datos astronómicos y con los datos provenientes de búsquedas directas e indirectas. En este sentido, el LHC y sus experimentos ATLAS y CMS juegan un papel fundamental en la solución de este problema.

ATLAS y CMS están localizados en los puntos 1 y 5 del acelerador LHC. En estos puntos, denominados puntos de interacción, haces de protones se cruzan, produciendo colisiones cuyos productos son detectados por estos dos experimentos. Los dos haces, que circulan en direcciones opuestas en el anillo de $27 \mathrm{~km}$, están compuestos, cada uno de ellos, por una serie de paquetes de protones (proton bunches). La energía de los protones, durante la primera corrida fue inicialmente de $3.5 \mathrm{TeV}$ y posteriormente de $4 \mathrm{TeV}$, resultando en energías de centro de masa de 7 y $8 \mathrm{TeV}$ respectivamente; los cruces de paquetes en los puntos de interacción sucedieron cada 50 ns. Para la segunda corrida, programada para iniciar en la primavera de 2015, se espera llegar a una energía de centro de masa de 13.5 a $14 \mathrm{TeV}$, con cruces de paquetes cada 25 ns. Con esta energía, y una mayor luminosidad (número de partículas por unidad de área y por unidad de tiempo en los puntos de interacción) se podrá escudriñar toda una nueva región cinemática en la búsqueda de nueva física.

Los detectores ATLAS y CMS rodean el tubo por donde circulan los dos haces de protones y están centrados en sus respectivos puntos de interacción. Estos detectores poseen una simetría cilíndrica y varias capas de detectores. Moviéndose hacia fuera desde el punto de interacción, los sistemas de detección que las partículas encuentran en su camino son:

- Sistema de reconstrucción de trazas (Tracker)

Es el detector más cercano al punto de interacción y está instalado justo alrededor del tubo del acelerador. Típicamente está construido a partir de detectores de pixeles y de micro-cintas de silicio y posee una alta resolución espacial. La tarea de este sistema es reconstruir las trayectorias de todas las partículas cargadas eléctricamente que emergen del punto de colisión.

- Calorímetro Electromagnético (ECAL)

Está construido a partir de materiales densos que logran detener y absorber los electrones, positrones y gammas provenientes del punto de interacción. Este sistema mide la cantidad de energía de estas partículas.

- Calorímetro Hadrónico (HCAL)

Similar al calorímetro electromagnético. Su propósito es detener y absorber los hadrones que atraviezan el calorímetro electromagnético, midiendo su energía.

- Sistema de muones ( $\mu$-System)

Localizado en la parte exterior del detector. $\mathrm{Su}$ propósito es reconstruir las trazas de los muones producidos en la colisión. Los muones son muy penetrantes y son las únicas partículas conocidas, con la excepción de los neutrinos, que logran atravesar las capas internas de detección. Al ser partículas cargadas, van a dejar trazas en estos detectores. 


\begin{tabular}{|c|l|}
\hline \hline Partículas & Sistemas de Detección \\
\hline \hline & \\
$\gamma$ & ECAL \\
$e^{ \pm}$ & Tracker + ECAL \\
$\mu^{ \pm}$ & Tracker + $\mu$-System \\
$\pi^{ \pm}$ & Tracker + ECAL + HCAL \\
$\mathrm{K}^{ \pm}$ & Tracker + ECAL + HCAL \\
$p$ & Tracker + ECAL + HCAL \\
$n$ & ECAL + HCAL \\
\hline
\end{tabular}

Cuadro II: Partículas detectables y los principales sistemas de detección involucrados.

Además de los sub-sistemas de detección, se cuenta con una configuración de campos magnéticos que permiten identificar las diferentes partículas y sus cargas eléctricas, a partir de la curvatura de sus trayectorias. En el caso de ATLAS, se cuenta con un magneto solenoidal rodeando el tracker, y una serie de magnetos alrededor de los detectores de muones, produciendo un campo toroidal en esta región. En el caso de CMS, se cuenta con un magneto solenoidal mucho más grande que rodea el tracker y los calorímetros. El campo magnético que emerge del solenoide es compactificado por un enorme yugo de hierro, donde se encuentran instalados los detectores de muones. En la Figura 8 se presenta una versión esquemática del detector CMS.

Las únicas partículas que pueden ser detectadas directamente en este tipo de experimentos son aquellas cuyos tiempos de vida son suficientemente largos como para que logren avanzar, al menos unos cuantos centímetros. Además, se requiere que estas partículas interactúen electromagnética o fuertemente, para que dejen rastro de su paso por los detectores. En el Cuadro II se presenta un resumen de las partículas detectables y de los sistemas de detección involucrados. Cualquier otra partícula no es detectable directamente, ya sea porque posee una vida media muy corta, por ejemplo $\tau^{ \pm}$, o porque solo interactúa débilmente; por ejemplo, los neutrinos.

Una colisión protón-protón a altas energías es en realidad una colisión entre partones, es decir, entre quarks y gluones al interior de los protones. Solamente un partón de uno de los protones tendrá una colisión dura con otro partón del otro protón. Los demás partones actuarán como espectadores y, como resultado de la colisión, quedaran en un estado de excitación tal que, en su proceso de desexcitación, emitirán partículas de bajo momento en todas direcciones; lo que se conoce como evento subyacente. Por el contrario, los dos partones que causaron la colisión dura generarán partículas con alto momento en la dirección transversal (alto $p_{T}$ ).

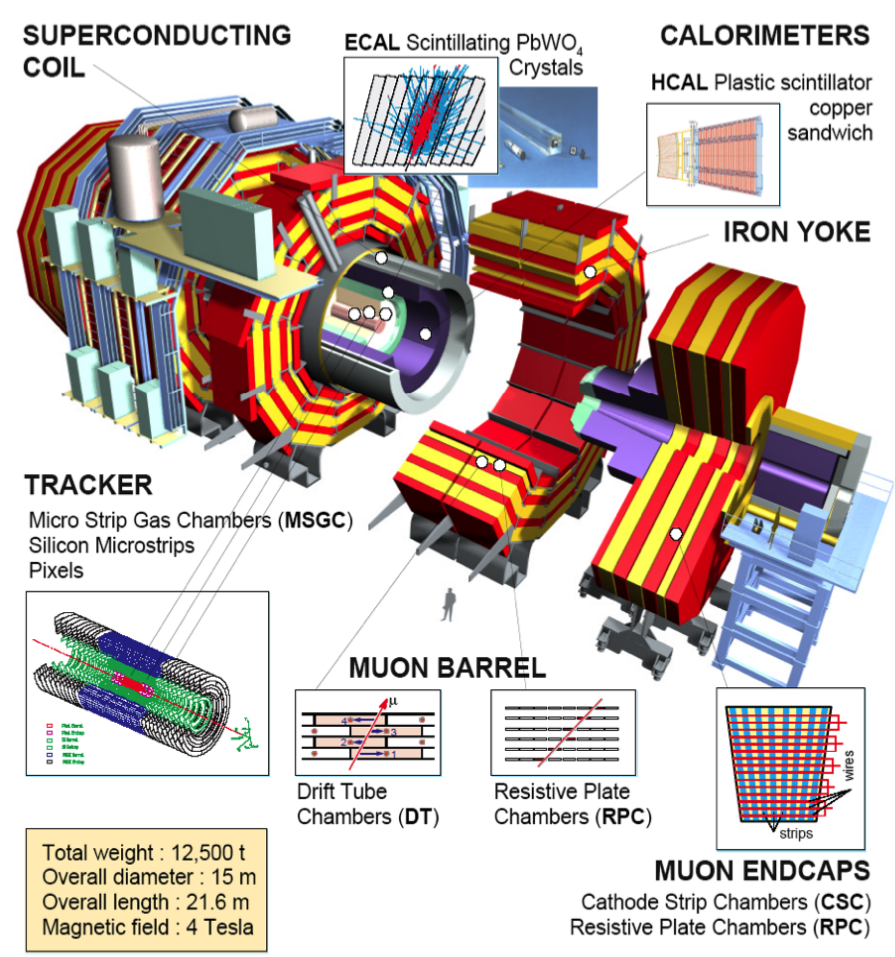

Figura 8: Diagrama esquemático del detector CMS con sus diferentes componentes (imagen tomada de [112]).

Cuáles partones participan en la colisión dura, y con qué fracción de momento lo hacen, es una cuestión de probabilidades que está cuantificada en lo que se conoce como: funciones de distribución partonica (pdf, parton distribution function). Estas funciones son distribuciones de probabilidad que indican cúal es la probabilidad de que, dado un tipo de partón, este posea cierta fracción $x$ del momento total de protón al cual pertenece.

En cada cruce entre paquetes de protones en los puntos de interacción del LHC, se producen múltiples colisiones protón-protón (del orden de 20 en la primera corrida y aproximadamente el doble en la segunda corrida). Una de cada billón de colisiones tendrá una interacción dura, partón-partón. Solo estas interacciones pueden contener física interesante para experimentos como ATLAS y CMS. En este tipo de colisiones se producen partones, en el estado final, con altos valores de $p_{T}$ que, al interactuar con el campo fuerte presente en el medio, desencadenan un proceso conocido como hadronización, en el cual se emiten chorros de partículas en una configuración cónica, conocidos como jets. Un jet es, por tanto, la señal experimental de la producción de un partón de alto momento en la colisión. La presencia de jets con alto $p_{T} \mathrm{y} / \mathrm{o}$ muones con alto $p_{T}$, son las principales señales que usan los experimentos para filtrar este tipo de eventos y para estudiarlos. 
Cuando se producen quarks b como resultado de una colisión, ellos forman hadrones con vidas medias suficientemente largas como para avanzar unos cuantos micrómetros, antes de decaer en forma de jets. Detectores modernos como ATLAS y CMS, están en capacidad de distinguir estos jets con vértices desplazados, e identificarlos como b-jets. Un caso similar es el de los $\tau$, que en un $65 \%$ de las veces decaen en forma hadrónica, produciendo jets de partículas con una topología especial, que permite distinguirlos, con cierta eficiencia, de otros jets $(\tau$-jets). Si un $\tau$ es muy energético, también puede producir un jet con vértice desplazado, debido al fenómeno de dilatación temporal de su vida media.

Por último, tenemos los neutrinos, las otras partículas del SM con vidas medias suficientemente largas (probablemente infinitas) como para ser detectadas. El problema con los neutrinos radica en que solo interactúan débilmente y que por tanto, solo pueden ser detectados con muy baja eficiencia en gigantescos detectores especialmente diseñados para este propósito. La probabilidad de que un neutrino deje una señal en los detectores de ATLAS o de CMS es prácticamente cero. Lo que sí pueden medir estos experimentos es el faltante que los neutrinos dejan en el balance final de energía de las colisiones. La medición de la cantidad de energía producida en el estado final de una colisión, corre por cuenta del sistema de calorímetros. Este sistema está segmentado angularmente en un número discreto de celdas con valores medios de ángulo polar y ángulo azimutal: $\theta_{n}, \phi_{n}$. A partir de la información contenida en el sistema de calorímetros se pueden construir cantidades experimentales como la Energía Transversal Faltante, $\mathbb{E}_{T}$ (Missing $\mathrm{E}_{T}$ ). Esta cantidad está definida como:

$$
\mathbf{E}_{T}=-\sum_{n}\left\{E_{n} \sin \theta_{n} \cos \phi_{n} \hat{i}+E_{n} \sin \theta_{n} \sin \phi_{n} \hat{j}\right\}
$$

donde $E_{n}$ corresponde a la energía depositada en la celda $\left(\theta_{n}, \phi_{n}\right)$ del sistema de calorímetros. Si esta cantidad es diferente a cero es porque hay un faltante de energía en el estado final de la colisión. La información vectorial de $\mathbf{E}_{T}$ nos indica en qué dirección, en promedio, escapó esa energía. Definiciones más sofisticadas de esta cantidad incluyen toda la información del tracker y del sistema de muones. Eventos con neutrinos en el estado final van a tener $\mathbf{E}_{T}$ diferente a cero.

Regresando a las búsquedas de materia oscura, la producción de WIMPs en el LHC debería ser resultado de colisiones duras con presencia de jets de alto $p_{T}$. Dado que los WIMPs solo interactúan débilmente, se comportarían como neutrinos muy masivos, es decir deberían producir altos valores de $\mathbb{E}_{T}$. Por ejemplo, en el caso de producción de materia supersimétrica que conserve la paridad $R$, cualquier pareja de partículas SUSY, producidas como resultado de la colisión dura, debería generar cadenas de decaimientos que terminen en un par de $\tilde{\chi}_{1}^{o}$. Las cadenas de decaimientos producirían jets de alto $p_{T}$ y en muchas ocasiones leptones de alto $p_{T}$. Los $\tilde{\chi}_{1}^{o}$ finales se manifestarían en forma de altos valores de $\mathrm{E}_{T}$.

Durante las corridas de 2011 y de 2012 en el LHC, la señal básica en las búsquedas iniciales de materia supersimétrica, correspondió a eventos con valores altos de $\mathbb{E}_{T}$ $\mathrm{y}$ jets de alto $p_{T}$. Hasta el momento, los resultados han sido negativos. Pero aún hay datos por analizar y estrategias más sofisticadas para llevar a cabo estas búsquedas.

\section{BÚSQUEDAS DE MATERIA OSCURA EN CANALES FUERTES}

Las búsquedas iniciales de SUSY, con conservación de la paridad $R$, llevadas acabo por los experimentos ATLAS y CMS, estuvieron enfocadas en canales de producción fuertes. Estos canales deberían poseer secciones transversales altas debido, precisamente, al carácter fuerte del mecanismo de producción. En colisiones protónprotón a energías de 8 o $14 \mathrm{TeV}$, los canales de producción dominantes son: la fusión de gluones, en el cual un gluón de uno de los protones interactúa con un gluón del otro protón para producir pares de gluinos o de s-quarks; y la aniquilación quark-antiquark, en la cual se producen pares de s-quarks, como se muestra en la Figura 9. Cada una de las partículas SUSY producidas debería generar una cadena de decaimientos independiente que, en general, involucra jets, leptones cargados, neutrinos y neutralinos. La topología del estado final de un evento de este tipo puede ser bastante compleja, como se muestra en la Figura 10. Varias conclusiones se pueden inferir a partir de esta figura:

- Las dos cadenas de decaimientos tienden a formar hemisferios, en lo que a la detección de partículas secundarias se refiere.

- Estos hemisferios se van a sobrelapar, debido a desbalances de momento entre los partones iniciales y a la presencia de $\mathrm{E}_{T}$ en el estado final.

- La presencia de varias partículas neutras que solo interactúan débilmente (neutrinos y neutralinos), hace imposible la reconstrucción completa del evento a nivel cinemático.

- La energía transversal faltante, $\mathbb{E}_{T}$, puede ser resultado de la presencia de neutrinos y de neutralinos.

- La energía de umbral para la producción de estos eventos debe ser mayor que la masa de los dos gluinos o de los dos s-quarks.

Si la masa de los gluinos y de los s-quarks es muy alta, este tipo de eventos quedan suprimidos a nivel cinemático. La energía de centro de masa del sistema partón-partón no es suficiente como para producir las 


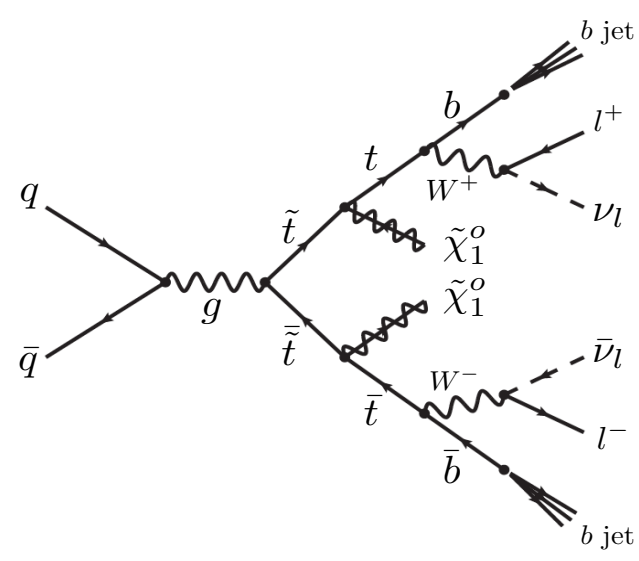

Figura 9: Producción de un par de s-tops y su decaimientos en neutralinos.

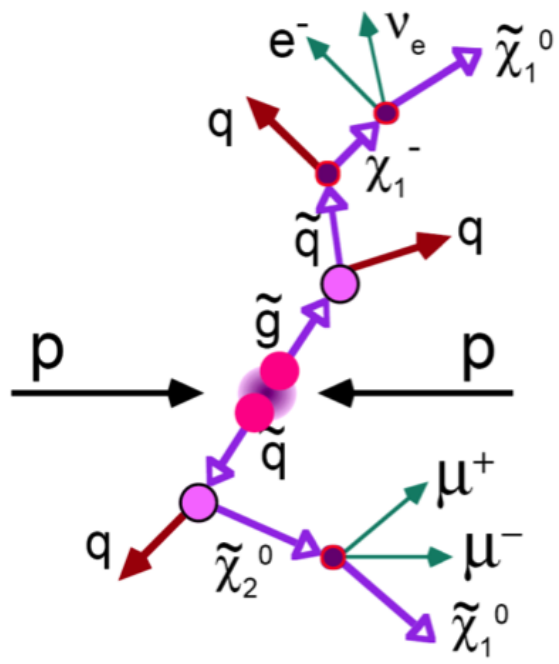

Figura 10: Topología del estado final de una colisión protónprotón en la que se produjeron un s-quark y un gluino (imagen tomada de [112]).

partículas SUSY iniciales. Pero, este hecho también previene que se produzcan partículas SUSY más ligeras como resultado de las cadenas de decaimiento. Todo el espectro de partículas SUSY cuyas masas sean menores que las de los gluinos o s-quarks, queda excluido. Por supuesto, la producción de los neutralinos, $\tilde{\chi}_{1}^{o}$, también queda excluida, ya que ellos son las partículas SUSY más ligeras. Las búsquedas de este tipo de eventos, llevadas a cabo por ATLAS y por CMS, con los datos a $8 \mathrm{TeV}$, han puesto limites inferiores a las masas de s-quarks y de gluinos del orden de $1.5 \mathrm{TeV}[114,115]$. Esto quiere decir que, para producir pares de estas partículas se requerirían más de $3 \mathrm{TeV}$ en el centro de masa del sistema partón-partón. En el caso de colisiones a $8 \mathrm{TeV}$, solo una fracción de los $4 \mathrm{TeV}$ que cada protón posee, es transportada por el partón que participa en la colisión dura. La probabilidad de que sucedan colisiones partón-partón con energías de centro de masa mayores a $3 \mathrm{TeV}$, es muy pequeña.

En realidad, las búsquedas realizadas hasta el momento han establecido límites de exclusión elevados para las masas de los s-quarks asociados a las dos primeras familias de quarks. Aún existe la posibilidad de que, por ejemplo, los s-tops tengan una masa por debajo de 1.5 $\mathrm{TeV}$, y de que su señal esté presente en los datos a 8 TeV. El s-top debería ser el s-quark más ligero al estar asociado al quark más pesado, el quark top. El canal de decaimiento más sencillo para el s-top es: $\tilde{t} \rightarrow t+\tilde{\chi}_{1}^{o}$, que implica que $m_{\tilde{t}}>m_{t}+m_{\tilde{\chi}_{1}^{o}}$. Las señales de la producción de pares $\tilde{t} \overline{\tilde{t}}$ son muy similares a las señales de producción de pares $t \bar{t}$, salvo por la presencia de mayor energía transversal faltante en el primer caso. Si los valores de $\mathbb{E}_{T}$ no son suficientemente grandes, y la sección transversal de producción es relativamente pequeña, es muy difícil separar la señal de $\tilde{t} \overline{\tilde{t}}$ del ruido de $t \bar{t}$. El problema se hace aún más difícil si $m_{\tilde{t}} \sim m_{t}$. En este caso $m_{\tilde{\chi}_{1}^{o}}$ es muy pequeña y $\mathbb{E}_{T} \simeq 0$, no pudiéndose usar esta variable para discriminar entre la señal y el ruido.

Si bien la búsqueda de s-tops en los datos existentes es muy difícil, vale la pena llevarla a cabo, ya que, como hemos mencionado, no se han establecido límites de exclusión para la masa de estas partículas. Recientemente, varios análisis que tratan de explotar estados finales y circunstancias cinemáticas diversas han sido propuestos y están siendo ejecutados $[116,117]$. Hasta el momento, no se han reportado resultados positivos.

\section{BÚSQUEDAS DE MATERIA OSCURA EN CANALES ELECTRODÉBILES}

El problema con las búsquedas de materia oscura supersimétrica en canales fuertes es que hay que producir partículas con carga de color (s-quarks y gluinos) para que ellas decaigan en neutralinos. Como mencionamos en la sección anterior, si estas partículas son muy masivas o difíciles de identificar, la presencia de materia oscura queda escondida en los datos. La otra opción es recurrir a canales de producción electrodébiles. En este tipo de canales, las partículas mediadoras del proceso no requieren carga de color, al igual que las partículas SUSY producidas. Todas ellas provienen del sector electrodébil del MSSM: bosones vectoriales, charginos y neutralinos. En general, los charginos y neutralinos son partículas más ligeras que los s-quarks y los gluinos. Por tanto, su producción directa está favorecida cinemáticamente y la posibilidad de encontrar señales en los datos existentes no está excluida. 
El mecanismo de producción electrodébil involucra la emisión de bosones vectoriales, $W^{ \pm}$o $Z^{o}$, por parte de los quarks que participan en la colisión dura. Estos bosones vectoriales se fusionan para producir partículas, del SM, o del sector SUSY, como se muestra en la Figura 11 para la producción de un par de $\tilde{\chi}_{1}^{o}$. Después de radiar los bosones vectoriales, los dos quarks pasan por el proceso de hadronización, convirtiéndose en dos jets de alto momento, producidos a ángulos pequeños $\left(j_{f}\right.$, forward jets). El resto del evento va a resultar en un estado final con partículas de alto $p_{T}$ que típicamente van a ser emitidas a grandes ángulos. En resumen, la estructura cinemática del estado final de eventos producidos por fusión de bosones vectoriales (VBF, Vector Boson Fusion) es la siguiente:

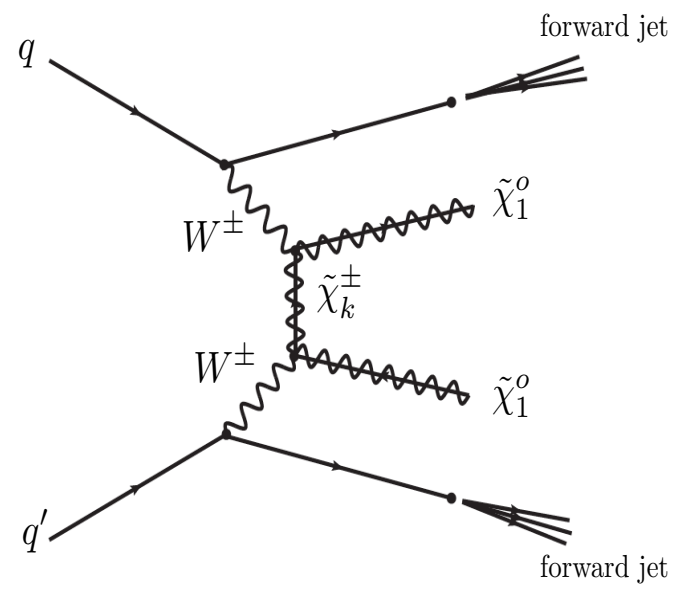

Figura 11: Producción electrodébil de un par de neutralinos acompañados por dos jets emitidos a ángulos pequeños (forward jets).

- Dos jets de alto momento, producidos a ángulos pequeños y en direcciones opuestas (forward jets)

- Partículas de alto momento transversal producidas a ángulos grandes.

- Una región, comprendida entre los ángulos pequeños y los ángulos grandes, en la que no hay presencia de partículas (pseudorapidity gap).

Estas señales cinemáticas permiten separar eventos producidos como resultado de $\mathrm{VBF}$, de eventos producidos como resultado de la interacción fuerte. Esto es importante por varias razones, siendo la primera el hecho de que el ruido producido por eventos de interacción fuerte, propios del SM, suele ser enorme. Al exigir la presencia de dos $j_{f}$ en el evento, sumado a la presencia de gaps de pseudorapidez, se elimina la gran mayoría de este ruido. Otra razón es que el mecanismo de producción electrodébil es más sencillo de calcular, y produce una topología de estado final mucho más simple, como se puede ver al comparar la Figura 11 con la Figura 9.

El problema con la producción de materia SUSY, o de materia oscura, por medio de mecanismos de VBF es que las secciones transversales son muy pequeñas, dado que los procesos son electrodébiles. Sin embargo, dadas las circunstancias actuales con los datos del LHC a 8 $\mathrm{TeV}$, en los cuales no se ha encontrado ninguna señal de física BSM, es necesario, e importante, explorar este tipo de canales, que no están excluidos cinemáticamente, y que son muy prometedores. Varias búsquedas en canales de VBF han sido propuestas [118, 119], y están siendo llevadas a cabo por grupos de análisis al interior de las colaboraciones CMS y ATLAS. Por ejemplo, en CMS se están desarrollando análisis de datos en 12 canales de VBF diferentes. Hasta el momento, no se han reportado resultados positivos.

Aún si no se encuentran evidencias de materia oscura en los datos de ATLAS y CMS a 8 TeV, en 2015 comenzará la segunda corrida del LHC a una energía de 13.5 o $14 \mathrm{TeV}$ (este valor aún no se ha determinado). Con esta energía de centro de masa, que es casi el doble de la de la primera corrida, se abren nuevamente amplias ventanas cinemáticas para buscar física BSM. Todas las herramientas de análisis de datos desarrolladas y refinadas durante la primera corrida, serán de la mayor utilidad con los nuevos datos. Por tanto, es de esperarse que muy pronto, después de iniciada la operación del LHC, ATLAS y CMS estén en capacidad de presentar resultados, ya sea positivos o negativos, de búsquedas de materia oscura en canales fuertes y electrodébiles.

\section{CONCLUSIONES}

Determinar la estructura microscópica de la materia oscura es, hoy por hoy, el problema más importante en Física de Partículas, Astrofísica y Cosmología. La evidencia astronómica que se ha recolectado en los últimos 80 años no deja mayores dudas respecto a su existencia. Los datos indican que esta materia es unas cinco veces más abundante que la materia que conocemos. Actualmente, hay pleno convencimiento de que las partículas constituyentes de la materia oscura tienen que ser de una naturaleza completamente nueva. Desde la década de 1980, múltiples experimentos han buscado estas partículas en forma directa e indirecta, o han tratado de producirlas en colisionadores de partículas. Actualmente, decenas de experimentos, de la más diversa índole continúan esta búsqueda en órbita alrededor de la Tierra, bajo tierra, bajo el mar, bajo el hielo polar, en grandes aceleradores de partículas, etc. El nivel de sofisticación al cual han llegado algunos de estos experimentos promete resultados concluyentes en los próximos años. El comienzo de la se- 
gunda corrida del acelerador LHC, en 2015, reanudará la búsqueda de materia oscura por parte de los experimentos ATLAS y CMS, bajo condiciones cinemáticas mucho más favorables. El sorprendente éxito de estos experimentos en la búsqueda del bosón de Higgs, durante la primera corrida del LHC, ha dejado claro que estos están en capacidad de descubrir cualquier rastro de física más allá del Modelo Estándar y que esté dentro del rango cinemático accesible, incluidas las partículas de materia oscura. Los próximos años prometen ser muy interesantes para esta área de la ciencia.

\section{AGRADECIMIENTOS}

Este artículo y la investigación científica asociada, desarrollada por el Grupo de Altas Energías de la Uni- versidad de los Andes, han sido posibles gracias al apoyo de COLCIENCIAS, del Departamento de Física y de la Facultad de Ciencias de la Universidad de los Andes. El autor agradece a Andrés Reyes, a Andrés Balaguera y a Andrés Flórez por sus comentarios al artículo, al igual que a Lina Duarte por su valiosa colaboración durante el proceso de redacción y de edición.
[1] S. Chatrchyan et al. [CMS Collaboration], Phys. Lett. B 716, 30 (2012) [arXiv:1207.7235 [hep-ex]].

[2] G. Aad et al. [ATLAS Collaboration], Phys. Lett. B 716, 1 (2012) [arXiv:1207.7214 [hep-ex]].

[3] F. Zwicky, Helv. Phys. Acta 6, 110 (1933).

[4] S. Smith, Astrophys. J. 83, 23 (1936).

[5] F. Zwicky, Astrophys. J. 86, 217 (1937).

[6] K. G. Begeman, A. H. Broeils and R. H. Sanders, Mon. Not. Roy. Astron. Soc. 249, 523 (1991).

[7] D. Zaritsky and S. D. M. White, Astrophys. J. 435, 599 (1994).

[8] M. Fich and S. Tremaine, Ann. Rev. Astron. Astrophys. 29, 409 (1991).

[9] C. S. Kochanek, Astrophys. J. 457, 228 (1996) [astro$\mathrm{ph} / 9505068]$.

[10] J. N. Bahcall, M. Schmidt and R. M. Soneira, Astrophys. J. 265, 730 (1983).

[11] J. A. R. Caldwell and J. P. Ostriker, Astrophys. J. 251, 61 (1981).

[12] S. Courteau, M. Cappellari, R. S. de Jong, A. A. Dutton, E. Emsellem, H. Hoekstra, L. V. E. Koopmans and G. A. Mamon et al., arXiv:1309.3276 [astro-ph.CO].

[13] A. J. Romanowsky, N. D. Douglas, M. Arnaboldi, K. Kuijken, M. R. Merrifield, N. R. Napolitano, M. Capaccioli and K. C. Freeman, Science 301, 1696 (2003) [astro-ph/0308518].

[14] R. Minchin, J. Davies, M. Disney, P. Boyce, D. Garcia, C. Jordan, V. Kilborn and R. Lang et al., Astrophys. J. 622, L21 (2005) [astro-ph/0502312].

[15] N. A. Bahcall and X.-h. Fan, Astrophys. J. 504, 1 (1998) [astro-ph/9803277].

[16] A. Kashlinsky, Phys. Rept. 307, 67 (1998) [astro$\mathrm{ph} / 9806236]$.

[17] D. Walsh, R. F. Carswell and R. J. Weymann, Nature 279, 381 (1979).

[18] J. A. Tyson, G. P. Kochanski and I. P. Dell'Antonio, Astrophys. J. 498, L107 (1998) [astro-ph/9801193].

[19] A. Choi, J. A. Tyson, C. B. Morrison, M. J. Jee, S. J. Schmidt, V. E. Margoniner and D. M. Wittman, Astrophys. J. 759, 101 (2012) [arXiv:1208.3904 [astroph.CO]].
[20] National Space Agency, NASA. Astronomy Picture of the Day. http://apod.nasa.gov/apod/ap100620.html

[21] O. Lahav, A. R. Liddle, Particle Data Group Reviews: Cosmological Parameters (2013).

[22] C. Alcock, D. R. Alves, A. Becker, D. Bennett, K. H. Cook, A. Drake, K. Freeman and M. Geha et al., Astrophys. J. 598, 597 (2003) [astro-ph/0308019].

[23] P. Tisserand et al. [EROS-2 Collaboration], Astron. Astrophys. 469, 387 (2007) [astro-ph/0607207].

[24] M. Milgrom, Astrophys. J. 270, 365 (1983).

[25] M. Milgrom, Astrophys. J. 270, 371 (1983).

[26] M. Milgrom, Astrophys. J. 270, 384 (1983).

[27] M. Reuter and H. Weyer, Int. J. Mod. Phys. D 15, 2011 (2006) [hep-th/0702051].

[28] D. Clowe, M. Bradac, A. H. Gonzalez, M. Markevitch, S. W. Randall, C. Jones and D. Zaritsky, Astrophys. J. 648, L109 (2006) [astro-ph/0608407].

[29] National Space Agency, NASA. Astronomy Picture of the Day. http://apod.nasa.gov/apod/ap060824.html

[30] K. A. Olive, D. N. Schramm, G. Steigman and T. P. Walker, Phys. Lett. B 236, 454 (1990).

[31] M. S. Smith, L. H. Kawano and R. A. Malaney, Astrophys. J. Suppl. 85, 219 (1993).

[32] T. P. Walker, G. Steigman, D. N. Schramm, K. A. Olive and H.-S. Kang, Astrophys. J. 376, 51 (1991).

[33] L. Bergstrom, Rept. Prog. Phys. 63, 793 (2000) [hep$\mathrm{ph} / 0002126]$.

[34] J. F. Beacom, N. F. Bell and S. Dodelson, Phys. Rev. Lett. 93, 121302 (2004) [astro-ph/0404585].

[35] S. Dodelson and L. M. Widrow, Phys. Rev. Lett. 72, 17 (1994) [hep-ph/9303287].

[36] E. W. Kolb, D. Lindley and D. Seckel, Phys. Rev. D 30, 1205 (1984).

[37] H.-C. Cheng, K. T. Matchev and M. Schmaltz, Phys. Rev. D 66, 036005 (2002) [hep-ph/0204342].

[38] L. J. Rosenberg and K. A. van Bibber, Phys. Rept. 325, $1(2000)$.

[39] C. Boehm, T. A. Ensslin and J. Silk, J. Phys. G 30, 279 (2004) [astro-ph/0208458].

[40] D. J. H. Chung, E. W. Kolb and A. Riotto, Phys. Rev. Lett. 81, 4048 (1998) [hep-ph/9805473]. 
[41] A. Birkedal-Hansen and J. G. Wacker, Phys. Rev. D 69, 065022 (2004) [hep-ph/0306161].

[42] A. Kusenko and M. E. Shaposhnikov, Phys. Lett. B 418, 46 (1998) [hep-ph/9709492].

[43] H. M. Hodges, Phys. Rev. D 47, 456 (1993).

[44] A. De Rujula, S. L. Glashow and U. Sarid, Nucl. Phys. B 333, 173 (1990).

[45] G. Shiu and L.-T. Wang, Phys. Rev. D 69, 126007 (2004) [hep-ph/0311228].

[46] J. A. R. Cembranos, A. Dobado and A. L. Maroto, Phys. Rev. Lett. 90, 241301 (2003) [hep-ph/0302041].

[47] J. R. Ellis, J. L. Lopez and D. V. Nanopoulos, Phys. Lett. B 247, 257 (1990).

[48] M. Drees, R. M. Godbole, P. Roy, Theory and Phenomenology of S-Particles, World Scientific (2004).

[49] P. Ramond, Field Theory: A Modern premier, Westview (1990).

[50] D. A. Dicus and V. S. Mathur, Phys. Rev. D 7, 3111 (1973).

[51] B. W. Lee, C. Quigg and H. B. Thacker, Phys. Rev. D 16, 1519 (1977).

[52] J. R. Ellis, hep-ph/0211168.

[53] G. Bertone, D. Hooper and J. Silk, Phys. Rept. 405, 279 (2005) [hep-ph/0404175].

[54] G. L. Kane, C. F. Kolda, L. Roszkowski and J. D. Wells, Phys. Rev. D 49, 6173 (1994) [hep-ph/9312272].

[55] L. Randall and R. Sundrum, Nucl. Phys. B 557, 79 (1999) [hep-th/9810155].

[56] G. F. Giudice, M. A. Luty, H. Murayama and R. Rattazzi, JHEP 9812, 027 (1998) [hep-ph/9810442].

[57] A. H. Chamseddine, R. L. Arnowitt and P. Nath, Phys. Rev. Lett. 49, 970 (1982).

[58] M. Dine and W. Fischler, Phys. Lett. B 110, 227 (1982).

[59] C. R. Nappi and B. A. Ovrut, Phys. Lett. B 113, 175 (1982).

[60] L. Alvarez-Gaume, M. Claudson and M. B. Wise, Nucl. Phys. B 207, 96 (1982).

[61] D. E. Kaplan, G. D. Kribs and M. Schmaltz, Phys. Rev. D 62, 035010 (2000) [hep-ph/9911293].

[62] Z. Chacko, M. A. Luty, A. E. Nelson and E. Ponton, JHEP 0001, 003 (2000) [hep-ph/9911323].

[63] A. G. Delannoy, B. Dutta, A. Gurrola, W. Johns, T. Kamon, E. Luiggi, A. Melo and P. Sheldon et al., Phys. Rev. Lett. 111, 061801 (2013) [arXiv:1304.7779 [hep$\mathrm{ph}]$.

[64] O. Lahav, A. R. Linddle. The Cosmological Parameters. Particle Data Group (2013). http://pdg.1bl.gov/2013/reviews/

[65] A. G. Sanchez, E. A. Kazin, F. Beutler, C.-H. Chuang, A. J. Cuesta, D. J. Eisenstein, M. Manera and F. Montesano et al., arXiv:1303.4396 [astro-ph.CO].

[66] E. W. Kolb and M. E. Turner, The Early Universe, Addison-Wesley (1990).

[67] G. Jungman, M. Kamionkowski and K. Griest, Phys. Rept. 267, 195 (1996) [hep-ph/9506380].

[68] M. W. Goodman and E. Witten, Phys. Rev. D 31, 3059 (1985).

[69] A. K. Drukier and L. Stodolsky, In *La Plagne 1984, Proceedings, Massive Neutrinos In Astrophysics and In Particle Physics*, 395

[70] A. K. Drukier, K. Freese and D. N. Spergel, Phys. Rev. D 33, 3495 (1986).

[71] L. Baudis, Phys. Dark Univ. 1, 94 (2012) [arXiv:1211.7222 [astro-ph.IM]].
[72] The CDMS Experiment http://cdms. berkeley.edu/

[73] The Soudan Underground Laboratory http://www. soudan. umn.edu/

[74] The CRESST Experiment http://www.cresst.de/exp_overview.php

[75] Laboratori Nazionali del Gran Sasso http://www.lngs.infn.it/

[76] The EDELWEISS Experiment http://edelweiss.in2p3.fr/

[77] Laboratoire Souterrain de Modane http://www-lsm.in2p3.fr/

[78] CoGeNT Dark Matter Experiment http://cogent.pnnl.gov/

[79] The Texono Experiment http://www.nu.to.infn.it/exp/all/texono/

[80] The ZEPLIN-III Experiment http://www.hep.ph.ic.ac.uk/ZEPLIN-III-Project/

[81] The Boulby Underground Laboratory http: //www.stfc.ac.uk/Boulby/default.aspx

[82] The XENON Dark Matter Project http: //xenon. astro.columbia.edu/

[83] Th XMASS Experiment http://www-sk.icrr.u-tokyo.ac.jp/xmass/index-e.html

[84] The Kamioka Observatory http://www-sk.icrr.u-tokyo.ac.jp/index-e.html

[85] The LUX Experiment http://lux.brown.edu/LUX_dark_matter/Home.html

[86] The DAMA Project http://people.roma2.infn.it/ dama/web/home.html

[87] The ANAIS Experiment http://gifna.unizar.es/anais/

[88] Laboratorio Subterráneo de Canfranc http://www.lsc-canfranc.es/es/

[89] The DM-Ice Project http://astro.fnal.gov/projects/DarkMatter/ DMice_project.html

[90] The PICASSO Project http://www.picassoexperiment.ca/

[91] The Sudbury Neutrino Observatory http://www.snolab.ca/

[92] The COUPP Experiment http://www-coupp.fnal.gov/

[93] M. Felizardo, T. A. Girard, T. Morlat, A. C. Fernandes, A. R. Ramos and J. G. Marques, J. Phys. Conf. Ser. 375 (2012) 012011.

[94] Laboratoire Souterrain à Bas Bruit http://www.1sbb.eu/

[95] The MACRO Experiment http://www. lngs.infn.it/lngs_infn/index.htm?mainRecord

[96] Baksan Neutrino Observatory http://www.inr.troitsk.ru/eng/ebno.html

[97] Super-Kamiokande http: //www-sk.icrr.u-tokyo.ac.jp/sk/index-e.html

[98] Lake Baikal Neutrino Telescope http://baikalweb.jinr.ru/

[99] AMANDA II Project http: //amanda.uci.edu/

[100] The ANTARES Collaboration http://antares.in2p3.fr/

[101] NESTOR Institute for Astroparticle Physics http://www.inp.demokritos.gr/nestor/

[102] IceCube, South Pole Neutrino Observatory https://icecube.wisc.edu/ 
[103] High Energy Stereoscopic System http://www.mpi-hd.mpg.de/hfm/HESS/

[104] Fermi Large Area Telescope http://www-glast.stanford.edu/

[105] C. Weniger, JCAP 1208, 007 (2012) [arXiv:1204.2797 [hep-ph]].

[106] The PAMELA Project http://pamela.roma2.infn.it/index.php

[107] The Alpha Magnetic Spectrometer http://home.web.cern.ch/about/experiments/ams

[108] M. Aguilar et al. [AMS Collaboration], Phys. Rev. Lett. 110, no. 14, 141102 (2013).

[109] O. Adriani et al. [ PAMELA Collaboration], Phys. Rev. Lett. 111, 081102 (2013) [arXiv:1308.0133 [astroph.HE]].

[110] ATLAS Experiment http://atlas.ch/

[111] ALICE, A Large Ion Collider Experiment http://aliceinfo.cern.ch/Public/Welcome.html

[112] The CMS Experiment http://cms.web.cern.ch/

[113] The LHCb Collaboration http://lhcb.web.cern.ch/lhcb/

[114] G. Aad et al. [ATLAS Collaboration], Phys. Rev. D 87, 012008 (2013) [arXiv:1208.0949 [hep-ex]].

[115] S. Chatrchyan et al. [CMS Collaboration], Phys. Rev. Lett. 109, 171803 (2012) [arXiv:1207.1898 [hep-ex]].

[116] CMS Supersymmetry Physics Results CMS Collaboration https://twiki.cern.ch/twiki/bin/view/CMSPublic/ PhysicsResultsSUS

[117] Summary Plots from the ATLAS Supersymmetry Physics Group

ATLAS Experiment

https://atlas.web.cern.ch/Atlas/GROUPS/PHYSICS/ CombinedSummaryPlots/SUSY/

[118] B. Dutta, A. Gurrola, W. Johns, T. Kamon, P. Sheldon and K. Sinha, Phys. Rev. D 87, 035029 (2013) [arXiv:1210.0964 [hep-ph]].

[119] A. G. Delannoy, B. Dutta, A. Gurrola, W. Johns, T. Kamon, E. Luiggi, A. Melo and P. Sheldon et al., Phys. Rev. Lett. 111, 061801 (2013) [arXiv:1304.7779 [hep$\mathrm{ph}]$. 